\title{
Super-Resolution Inversion of Non-Stationary Seismic Traces
}

\author{
Jinghuai Gao ${ }^{1, *}$, Hongling Chen ${ }^{1}$, Lingling Wang ${ }^{2}$ and Bing Zhang ${ }^{1}$ \\ ${ }^{1}$ School of Information and Communications Engineering, Faculty of Electronic and \\ Information Engineering, Xi'an Jiaotong University, Xi'an, Shaanxi, 710049, China. \\ 2 Institute of Geophysics and Geomatics, China University of Geosciences (Wuhan), \\ Wuhan, Hubei, 430074, China.
}

Received 6 July 2020; Accepted 18 December 2020

\begin{abstract}
In reflection seismology, the inversion of subsurface reflectivity from the observed seismic traces (super-resolution inversion) plays a crucial role in target detection. Since the seismic wavelet in reflection seismic data varies with the travel time, the reflection seismic trace is non-stationary. In this case, a relative amplitude-preserving super-resolution inversion has been a challenging problem. In this paper, we propose a super-resolution inversion method for the non-stationary reflection seismic traces. We assume that the amplitude spectrum of seismic wavelet is a smooth and unimodal function, and the reflection coefficient is an arbitrary random sequence with sparsity. The proposed method can obtain not only the relative amplitude-preserving reflectivity but also the seismic wavelet. In addition, as a by-product, a special $Q$ field can be obtained.

The proposed method consists of two steps. The first step devotes to making an approximate stabilization of non-stationary seismic traces. The key points include: firstly, dividing non-stationary seismic traces into several stationary segments, then extracting wavelet amplitude spectrum from each segment and calculating $Q$ value by the wavelet amplitude spectrum between adjacent segments; secondly, using the estimated $Q$ field to compensate for the attenuation of seismic signals in sparse domain to obtain approximate stationary seismic traces. The second step is the super-resolution inversion of stationary seismic traces. The key points include: firstly, constructing the objective function, where the approximation error is measured in $L_{2}$ space, and adding some constraints into reflectivity and seismic wavelet to solve ill-conditioned problems; secondly, applying a Hadamard product parametrization (HPP) to transform the non-convex problem based on the $L_{p}(0<p<1)$ constraint into a series of convex optimization problems in $L_{2}$ space, where the convex optimization problems are solved by the singular value decomposition (SVD) method and the regularization parameters are determined by the L-curve method in the case of single-variable inversion. In this paper, the effectiveness of the proposed method is demonstrated by both synthetic data and field data.
\end{abstract}

*Corresponding author. Email addresses: jhgao@xjtu.edu.cn (J. H. Gao), 859311743@qq.com (H. L. Chen), wangll@cug.edu.cn (L. L. Wang), 531810749@qq.com (B. Zhang) 
AMS subject classifications: 86A22

Key words: Non-stationary seismic trace, reflectivity inversion, super-resolution, attenuation compensation, $L_{p}$ regularization.

\section{Introduction}

In reflection seismology, recovering the subsurface reflectivity from the observed seismic data lies in the core of target exploration. To retrieve the reflectivity, super-resolution (someone called high-resolution) inversion methods are one of the mainstream approaches. Following the research of [1], the super-resolution inversion can be treated as a process to retrieve the fine-scale structures of an object from coarse-scale information only. It may be more reasonable to use the term "seismic super-resolution" instead of the term "seismic high-resolution". Broadly speaking, the seismic deconvolution [2,3], spectral whitening [4] and so on can be regarded as some kinds of super-resolution processing. In a narrow sense, the super-resolution inversion usually refers to the reflectivity inversion [5-7].

It should be pointed out that the convolution model is the theoretical basis of superresolution inversion. Before super-resolution inversion, therefore, it is necessary to compensate for the seismic data because the field seismic data is non-stationary with amplitude absorption and phase dispersion caused by intrinsic an-elasticity of subsurface media [8-10]. To accomplish this, the $Q$ values are required. Various methods have been proposed to estimate the $Q$ values [11-15], such as the methods in the Fourierfrequency domain and the time-frequency domain. In the Fourier-frequency domain, the approaches usually include the logarithm spectral ratio, centroid frequency shift, and peak frequency shift methods. All amplitude spectra need to be calculated within a time window. For the reflection seismic data, it is full of challenges to properly select the window function and window length. To adaptively select a proper window, [16] proposed a method to divide non-stationary seismic traces into several stationary segments adaptively, and then gave a method to estimation $Q$ values. In this work, we shall develop the method of [16].

The compensation methods of attenuation effects (inverse $Q$ filtering) can be applied to make the non-stationary seismic data stationary [17-19]. It is well known that the conventional inverse $Q$ filtering methods can boost noise and introduce numerical instability. To address this issue, many improved strategies have been proposed, one of which is implemented by the inverse problem framework [20]. [20] proposed a seismic absorption compensation method by the inversion scheme in a sparse domain. In this paper, we compensate for the attenuation effects by this inverse framework in the sparse domain.

After compensation, the non-stationary seismic data is turned into the stationary one. In the following parts, we review the super-resolution inversion based on the convolution 
model. Generally, the super-resolution inversion is ill-posed because of the band-limited wavelet and noise. The regularization method can be used as a feasible approach to address this issue. In general, the cost function is expressed as

$$
J=\min _{\mathbf{r}}\{f(\mathbf{r})+g(\mathbf{r})\}
$$

where $f(\mathbf{r})$ is a loss function, and $g(\mathbf{r})$ is a constraint function. The loss function $f(\mathbf{r})$ is usually selected as a square loss function. Based on the sparsity assumption of reflectivity, the constraint function is normally taken as the norm of the reflectivity, and the form of $g(\mathbf{r})$ is given by

$$
g(x)=\mu\|\mathbf{r}\|_{p}^{p}
$$

where $\mu$ denotes the regularization parameter, and $\|\mathbf{r}\|_{p}^{p}$ indicates the $L_{p}(0<p \leq 1)$ norm of variable $\mathbf{r}$. Theoretically, the $L_{0}$ regularization yields the sparsest solution [21]. However, it is full of challenges to solve the $L_{0}$ regularization, because it is a combinatory optimization problem [22]. Generally, a common alternative is to consider the $L_{1}$ regularization because it is convex and can be solved efficiently. In addition, most researchers apply $L_{1}$ regularization to implement the reflectivity inversion. For instance, [23] proposed a seismic sparse-spike deconvolution method to simultaneously recover wavelet and reflectivity, where authors imposed $L_{1}$ regularization to constrain the reflectivity.

Though the $L_{1}$ regularization has become so widespread, the $L_{1}$ regularization penalizes the larger coefficients more heavily than the smaller coefficients, unlike the more democratic penalization of the $L_{0}$ regularization [21], so that the $L_{1}$ regularization will cause the weak signal loss especially for noisy data. To address this issue, naturally, another consideration is the $L_{p}(0<p<1)$ regularization which outperforms the $L_{1}$ regularization [22,24-27]. The $L_{p}(0<p<1)$ regularization can achieve a sparser solution in contrast to the $L_{1}$ regularization [28]. Even though the $L_{p}(0<p<1)$ regularization leads to a non-convex optimization problem, it is easier to be solved than the $L_{0}$ regularization. Based on the advantages of the $L_{p}(0<p<1)$ regularization, the $L_{p}(0<p<1)$ regularization is applied to extract reflectivity from seismic traces in this paper.

To solve the $L_{p}(0<p<1)$ regularization, the algorithm and regularization parameter play crucial roles in the inversion results. Most $L_{p}(0<p<1)$ regularization algorithms in the literatures are somewhat opaque to researchers who are not well-versed in the theory of optimization, so that it's difficult to program. Therefore, to easily solve this inversion problem by developing the work of [29], we propose a stable and simple algorithm, called SVD-HPP [27], to solve the $L_{p}(0<p \leq 1)$ regularization, which can turn $L_{p}(0<p \leq 1)$ regularization into a series of $L_{2}$ regularizations. Then the existing algorithms can be applied to solve each $L_{2}$ regularization [30]. In this paper, the SVD method is adopted to solve each $L_{2}$ regularization for high inversion accuracy. Furthermore, the regularization parameter is easy to be selected because some conventional methods, such as L-curve method [31] and generalized cross-validation method (GCV) [32], are originally derived based on the $L_{2}$ regularization. Compared with the GCV method, the L-curve can obtain 
a better regularization parameter in most practical cases, which is applied to determine the regularization parameters in this paper.

Eq. (1.1) is only applicable to the case of known wavelet. If the wavelet is unknown, the alternating iterative inversion method is considered [7,23], because of the challenges to estimate an accurate seismic wavelet. According to the commutative law of convolution, the wavelet and reflectivity can be exchanged into the form of the forward matrix. Then the objective function containing two types of variables, i.e., the reflectivity and wavelet, is established, where the $L_{p}(0<p<1)$ norm is added to constrain the reflectivity and a mixed norm is used to constrain the seismic wavelet. Finally, the inversion problems are solved by alternating iteration and proximal splitting methods, where the SVD-HPP is applied to solve the sub-problem of reflectivity. In this section, the regularization parameters are determined by a manual operation like to the work of [7], because of the coupling of multiple variables.

In this paper, we establish a theoretical system of super-resolution inversion in a nonstationary frame, which is implemented by a two-stage method to extract reflectivity from non-stationary seismic data. The first stage is the stabilization of time-varying data. We first decompose the non-stationary seismic data into several segments, and then estimate a $Q$ value in each segment. Finally, the attenuation compensation method in a sparse domain can be applied to compensate for the non-stationary seismic data, so that the time-varying seismic data are transformed into time-invariant data. In the second stage, the super-resolution inversion method based on the $L_{p}(0<p<1)$ regularization is applied to invert the reflectivity under the condition of known wavelet. Moreover, the alternating iterative super-resolution inversion method is adopted to estimate the reflectivity and seismic wavelet simultaneously, where the $L_{p}(0<p<1)$ norm and mixed norm are added to constrain the reflectivity and wavelet, respectively. The $L_{p}(0<p<1)$ regularization in the objective functions can be solved by the SVD-HPP algorithm.

The structure of the paper is organized as follows: first, we elaborate on how to finish the stabilization of non-stationary seismic traces. Second, we introduce the reflectivity inversion method based on the $L_{p}(0<p<1)$ regularization, and determine the selection of $p$ in $L_{p}(0<p<1)$. Third, to avoid the effect of seismic wavelet, the alternating iterative inversion is proposed to recover the reflectivity and wavelet simultaneously. Finally, both synthetic and field examples are tested to demonstrate the effectiveness of the proposed method. In Appendix B, the SVD-HPP algorithm is derived.

\section{Method}

To obtain the credible reflectivity coefficients from non-stationary seismic traces, the first key point is to transform the non-stationary data to an approximately stationary one, and then the proposed super-resolution inversion method is applied to recover the reflectivity. The proposed main work-flow is shown in Fig. 1 which clearly shows the complete process to implement the super-resolution inversion of non-stationary seismic traces. The following sections present the corresponding theories. 


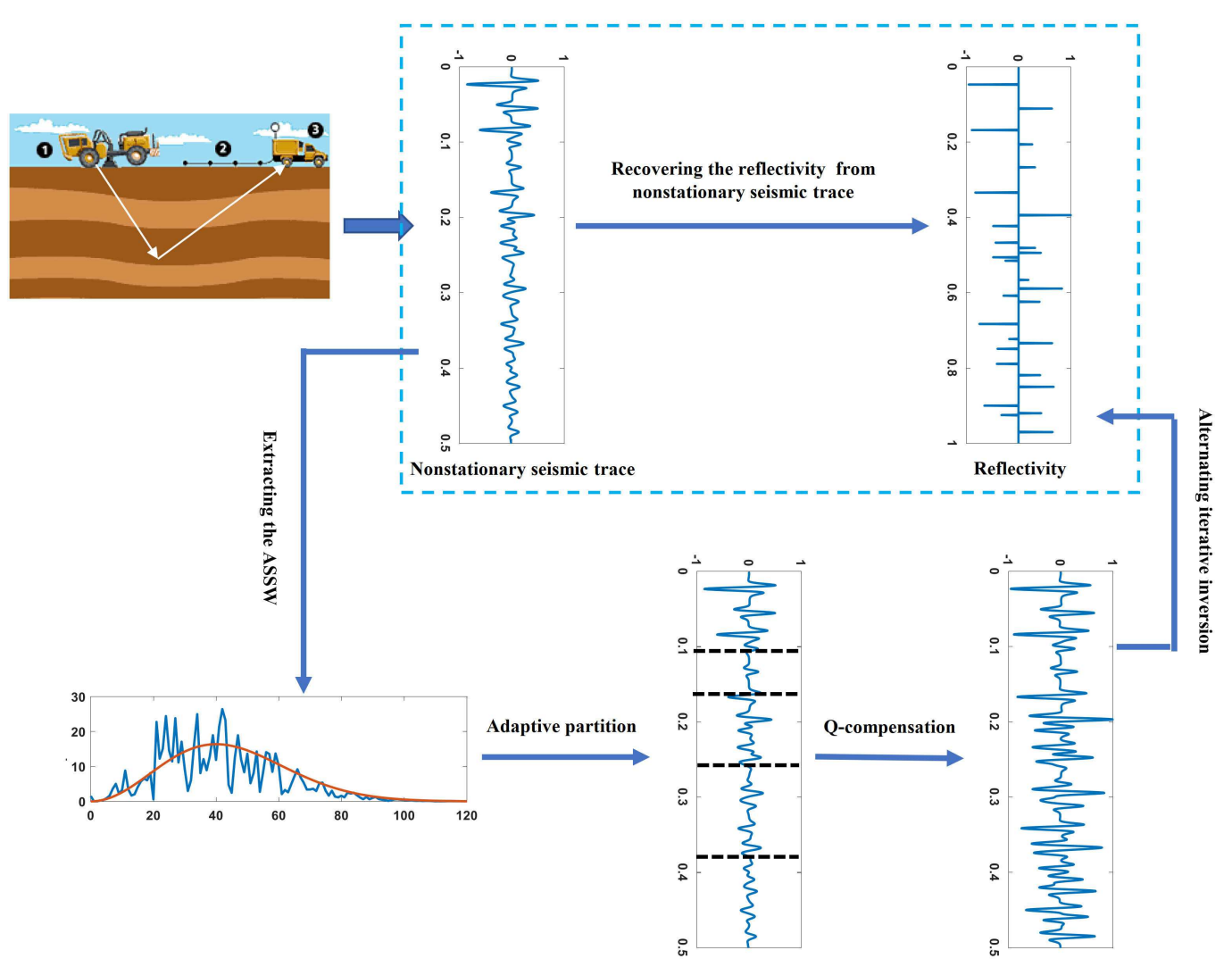

Figure 1: Work-flow of non-stationary super-resolution inversion.

\subsection{Stabilization of non-stationary seismic traces}

In a viscoelastic medium, it's full of challenges to enhance the resolution of seismic data because of the time-varying seismic wavelet. However, the seismic wavelet usually does not change quickly, which means that the amplitude of wavelet varies slowly, and it allows us to partition the non-stationary seismic trace into several stationary segments according to the similarity of wavelet amplitudes [16]. Then an equivalent $Q$ value can be estimated in each adaptive segment. Finally, the compensation method in the sparse domain is applied to eliminate the attenuation effect of seismic data [20]. For completeness and convenience, the following steps review the corresponding theories.

\section{Step 1: Extracting the amplitude spectrum of the seismic wavelet}

To divide the non-stationary seismic trace into several approximately stationary segments, how to extract the amplitude spectrum of a seismic wavelet (ASSW) is a key. In this paper, the method based on contraction operator mapping (COM method), proposed by [33], is applied to obtain the ASSW. The COM method can extract an accurate ASSW 
without whiteness assumption of reflectivity and setting beforehand function form. The algorithm of the COM method is listed in Algorithm 1.

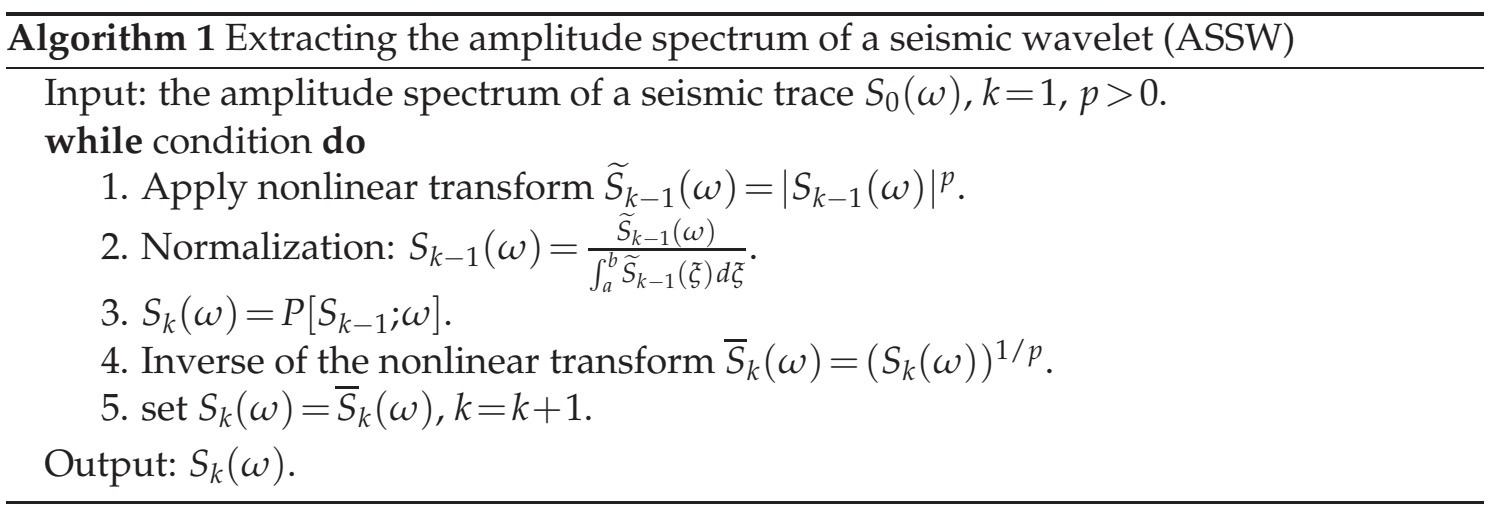

In Algorithm 1 , the $P[f ; x]$ indicates a mapping operator expressed as

$$
P[f ; x]=c_{p}\left(F_{p}(f ; x)\right)^{\alpha}\left(1-F_{p}(f ; x)\right)^{\beta},
$$

where $F_{p}\left(S_{k-1} ; \omega\right)=\int_{a}^{\omega} S_{k-1}(\xi) d \xi$ and $S_{k-1}(\xi)$ can be obtained by step 2 in Algorithm 1 . The effective frequency bandwidth of seismic trace is defined in $(a, b)$ with $0 \leq a<\omega<b$. Appendix A shows how to determine the parameters $c_{p}, \alpha$, and $\beta$.

To test the COM method, Fig. 2(a) displays a reflectivity that satisfies the Cauchy distribution. Fig. 2(b) shows a synthetic trace generated by convoluting a $30 \mathrm{~Hz}$ Ricker wavelet with the reflectivity shown in Fig. 2(a). Then the COM method is applied to extract the ASSW and the result is shown in Fig. 2(c). The estimated ASSW (black solid line) is almost the same as the true one (red dashed line) by only one iteration. Therefore, the COM method can be used to estimate an accurate ASSW.

\section{Step 2: Adaptive partition of non-stationary seismic trace}

Based on the work of [16], the same method is applied to divide the non-stationary seismic trace adaptively, except for the ASSW method. Firstly, we define an atom window function $g_{j}$ centering at the $j_{t h}$ sampling point, and a collection of atom windows satisfies a uniform partition of unity (POU) [34]. Secondly, a molecular-Gabor (MG) window is constructed by the summation of neighboring atom windows, which can be formulated as

$$
G_{k}(t)=\sum_{j=M_{k-1}+1}^{M_{k}} g_{j},
$$

where $M_{k-1}$ and $M_{k}$ indicate the indexes of the first and last atom window's center points for the $k_{t h}$ MG window which consists of $\left(M_{k}-M_{k-1}\right)$ atom windows. The index of the start point in the first MG window is $M_{0}$, which is equal to 0 . Thus, there is just the index 


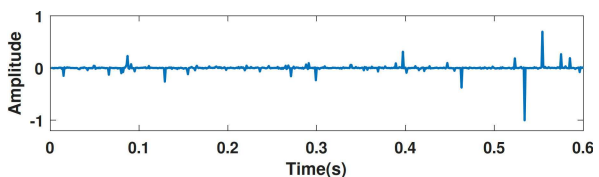

(a)

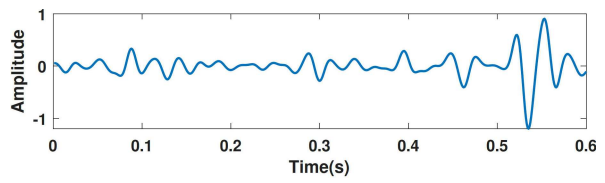

(b)

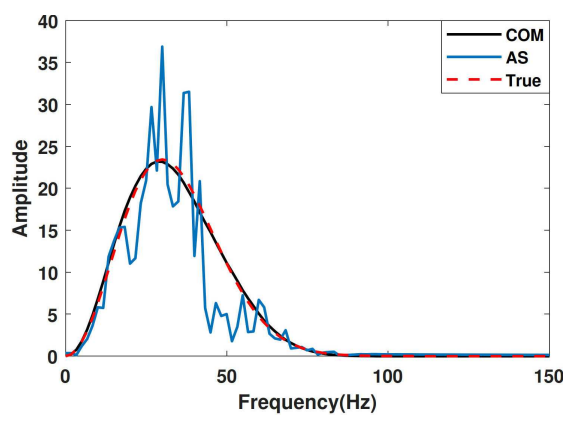

(c)

Figure 2: Extracting the amplitude spectrum. (a) the reflectivity satisfying the Cauchy distribution, (b) the corresponding synthetic trace, and (c) the extracted amplitude spectrum. The black solid line is the result obtained by the COM method, the red dotted line is the true ASSW, and the blue solid line is the amplitude spectrum (AS) of Fig. 2(b).

$M_{k}$ to be determined for the $k_{t h}$ MG window. To determine $M_{k}$ in the $k_{t h}$ MG window, the similarity of wavelet amplitude spectra is considered to establish an objective function

$$
J_{M_{k}}=\max _{M_{k}} \frac{<L_{0}, \widetilde{L}_{k}>}{\left\|L_{0}\right\|\left\|\widetilde{L}_{k}\right\|}+\zeta \frac{<L_{k-1}, \widetilde{L}_{k}>}{\left\|L_{k-1}\right\|\left\|\widetilde{L}_{k}\right\|},
$$

where $\|\cdot\|$ is the $L_{2}$ norm, and $L_{0}$ denotes a reference wavelet amplitude spectrum which can be obtained from well logs or shallow seismic data. $\widetilde{L}_{k}$ denotes the estimated ASSW in the undetermined $k_{t h} \mathrm{MG}$ window, i.e.,

$$
\widetilde{L}_{k}=\operatorname{COM}\left[s(t) \sum_{j=M_{k-1}+1}^{\widetilde{M}_{k}} g_{j}(t)\right],
$$

where the operator COM represents the COM method to extract ASSW, $s(t)$ is the nonstationary seismic trace, and $\widetilde{M}_{k}$ is the undetermined index of the $k_{t h} \mathrm{MG}$ window. Similarly, $L_{k-1}$ is the ASSW in the determined $(k-1)_{t h}$ MG window with known $M_{k-1}$. For the parameter $\zeta$ in Eq. (2.3), one can select that $\zeta=0.5$. By maximizing the function (2.3), the $k_{t h}$ MG window will be determined. Finally, one can implement the adaptive partition of non-stationary seismic trace using the determined MG windows as follows,

$$
\widetilde{s}_{k}(t)=s(t) \sum_{j=M_{k-1}+1}^{M_{k}} g_{j}(t),
$$

where $\widetilde{s}_{k}(t)$ is the $k_{t h}$ segment of non-stationary seismic trace $s(t)$. For each segment, some conventional methods can be applied to estimate $Q$ values, such as the spectral ratio [14], centroid frequency shift [35]. Here, the method proposed by [15] is applied to finish the equivalent $Q$-estimation. 


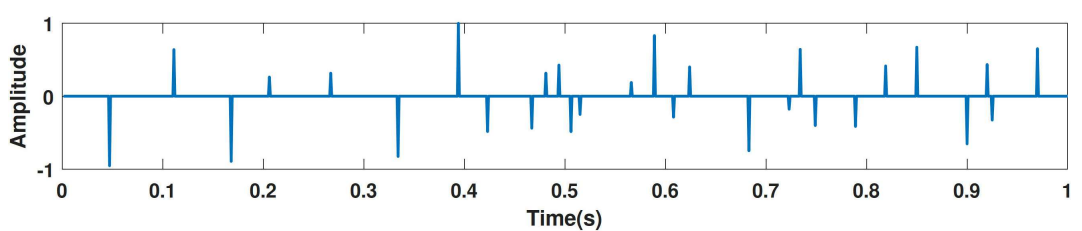

(a)

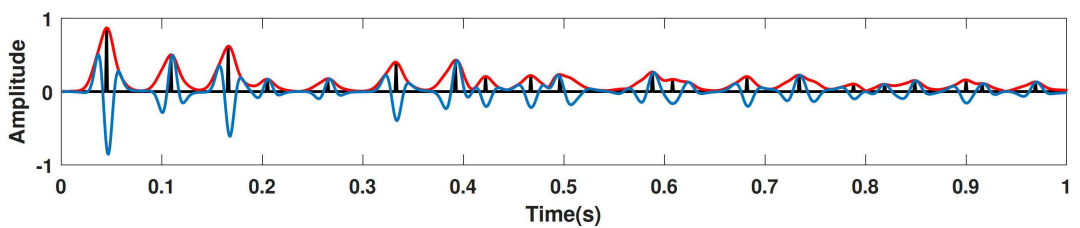

(b)

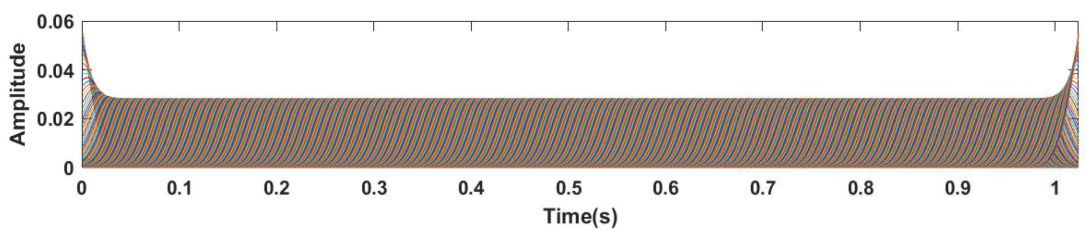

(c)

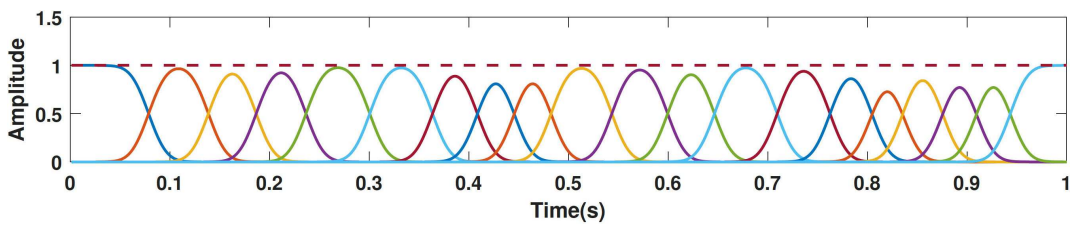

(d)

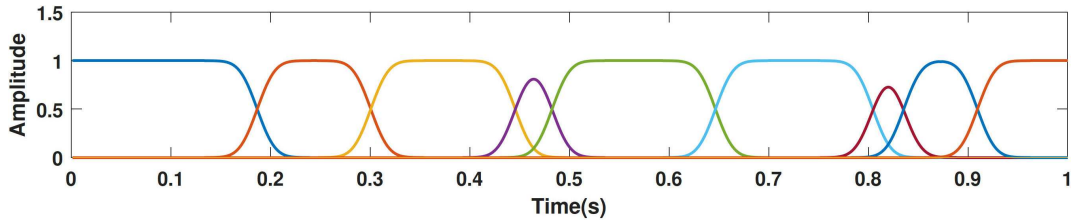

(e)

Figure 3: Construction of MG windows. (a) the true reflectivity model, (b) the corresponding synthetic nonstationary data with its envelope (red line) and envelope local peaks (black line), (c) the atom windows, (d) the initial MG windows, and (e) the final MG windows.

Fig. 3 illustrates the partition of a non-stationary seismic trace. Fig. 3(a) and Fig. 3(b) show the reflectivity and the corresponding non-stationary seismic trace, respectively. To partition the seismic data, the atom windows are firstly constructed, as shown in Fig. 3(c). Then, the initial MG windows are constructed using atom windows, as shown in Fig. 3(d). Finally, Fig. 3(e) shows the final MG windows constructed by the theory described above. 


\section{Step 3: Attenuation compensation method in the sparse domain}

Because of the inelastic attenuation of the subsurface medium, the seismic trace is nonstationary. In the time-frequency domain, the non-stationary seismic trace can be modelled as

$$
Y_{0}(\omega, \tau)=Y(\omega, \tau) \alpha(\omega, \tau),
$$

where $Y_{0}(\omega, \tau)$ represents the attenuated seismic data in the time-frequency domain and $Y(\omega, \tau)$ represents the $Q$-compensation seismic data in the time-frequency domain. The corresponding matrices of them are represented as $\mathbf{Y}_{0}$ and $\mathbf{Y} . \alpha(\omega, \tau)$ denotes the timefrequency attenuation factor based on the Kolsky-Futterman $Q$ model [36], written as

$$
\alpha(\omega, \tau)=e^{-\frac{\omega \tau}{2 Q(\tau)}} e^{i \ln \left|\frac{\omega}{\omega r}\right| \frac{\omega \tau}{\pi Q(\tau)}},
$$

where $\omega_{r}$ denotes the reference frequency, $Q(\tau)$ denotes the equivalent quality factor which is inversely proportional to the absorption effect of the subsurface medium. In Eq. (2.7), the first exponent indicates the amplitude attenuation, and the second describes the velocity dispersion and phase rotation. It's obvious Eq. (2.7) will be equal to one when the $Q(\tau)$ value is equal to infinity.

To overcome the instability of inverse $Q$ filtering, we regard Eq. (2.6) as an inverse problem. According to the work of [20], $Y_{0}(\omega, \tau)$ and $Y(\omega, \tau)$ can be treated as two timefrequency representations using the Synchrosqueezing transform. Then the objective function is constructed as

$$
J_{\widetilde{\mathbf{Y}}}=\min _{\widetilde{\mathbf{Y}}}\left\|\widetilde{\mathbf{Y}}_{0}-\widetilde{\Psi} \widetilde{\mathbf{Y}}\right\|_{2}^{2}+\mu\|\widetilde{\mathbf{Y}}\|_{1}
$$

where $\widetilde{\mathbf{Y}}=\operatorname{vec}(\mathbf{Y}), \widetilde{\mathbf{Y}}_{0}=\operatorname{vec}\left(\mathbf{Y}_{0}\right)$, and $\widetilde{\Psi}=\operatorname{diag}(\operatorname{vec}(\Psi))$. The operator vec can vectorize the two-dimensional (2D) data into a single column, the operator diag can transform a vector into a diagonal matrix, and the attenuation matrix $\Psi$ is represented as

$$
\Psi=\left[\begin{array}{cccc}
\alpha\left(\omega_{1}, \tau_{1}\right) & \alpha\left(\omega_{1}, \tau_{2}\right) & \cdots & \alpha\left(\omega_{1}, \tau_{q}\right) \\
\alpha\left(\omega_{2}, \tau_{1}\right) & \alpha\left(\omega_{2}, \tau_{2}\right) & \cdots & \alpha\left(\omega_{2}, \tau_{q}\right) \\
\vdots & \vdots & \vdots & \vdots \\
\alpha\left(\omega_{\rho}, \tau_{1}\right) & \alpha\left(\omega_{\rho}, \tau_{2}\right) & \cdots & \alpha\left(\omega_{\rho}, \tau_{q}\right)
\end{array}\right]
$$

where $\rho$ denotes the frequency sampling number and $q$ denotes the time sampling number. The iteratively reweighted least-squares algorithm is used to solve Eq. (2.8) [20].

Because the phase compensation is an inherently stable process, we just consider amplitude compensation which will cause instability and amplify noise. Fig. 4 shows the compensation results for the attenuated data with different $Q$ values in the noise-free case. Comparing with the conventional method without regularization, the attenuation compensation method in the sparse domain (ACSD) can compensate for the amplitude of attenuated data well without the amplification of numerical error. 

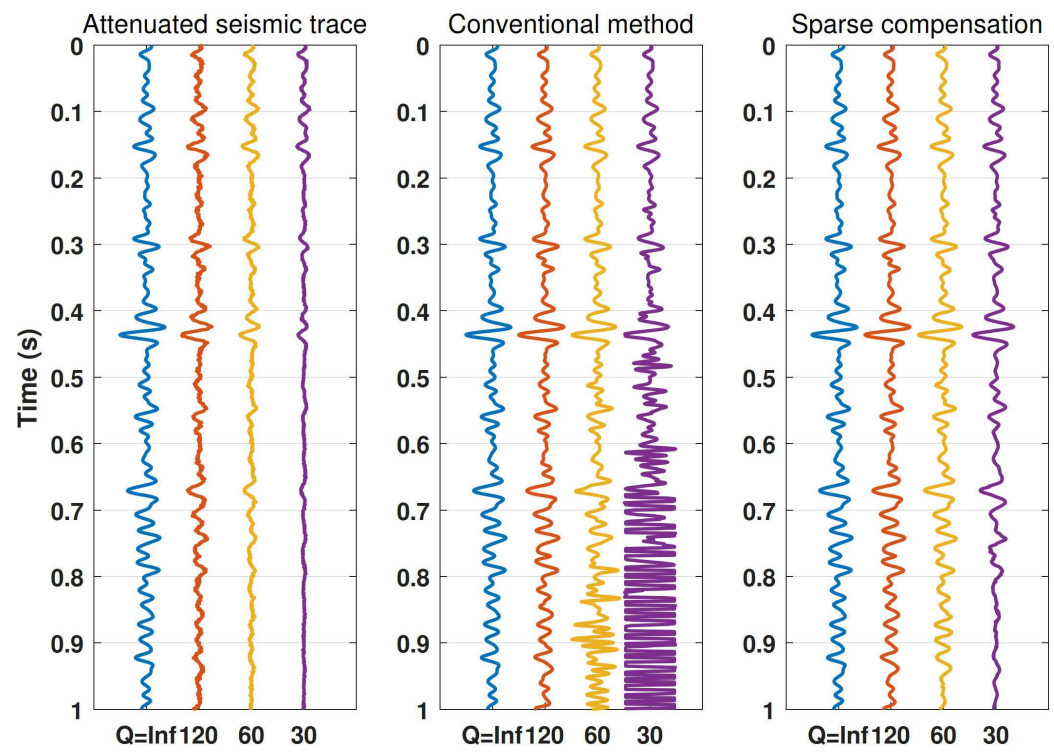

Figure 4: Attenuation compensation. The synthetic traces are generated by different $Q$ values, and the corresponding compensation results are calculated by the conventional method without regularization and the ACSD method.

\subsection{Super-resolution inversion based on the $L_{p}(0<p<1)$ regularization}

The conventional convolution model can be used to model the stationary seismic trace. Therefore, the seismic trace $y(t)$ after $Q$-compensation can be represented as

$$
y(t)=w(t) * r(t)+n(t),
$$

where $r(t)$ denotes the reflectivity containing the information of subsurface medium, $w(t)$ is the seismic wavelet, $n(t)$ denotes random noise, and $*$ indicates convolution operator. We write Eq. (2.10) into matrix form as follows

$$
\mathbf{y}=\mathbf{W r}+\mathbf{n},
$$

where $\mathbf{y}=\left[y\left(t_{1}\right), y\left(t_{2}\right), \cdots, y\left(t_{q}\right)\right]^{T}, \mathbf{r}=\left[r\left(t_{1}\right), r\left(t_{2}\right), \cdots, r\left(t_{q}\right)\right]^{T}$, and $\mathbf{n}=\left[n\left(t_{1}\right), n\left(t_{2}\right), \cdots, n\left(t_{q}\right)\right]^{T}$. $T$ denotes transposition. The convolution matrix $\mathbf{W}$ consists of seismic wavelet with $2 l+1$ sampling points $(2 l+1 \ll q)$ where $l$ refers to the half length of a wavelet. Considering a known seismic wavelet, the cost function $J_{\mathrm{r}}$ is established as

$$
J_{\mathbf{r}}=\min _{\mathbf{r}} \frac{1}{2}\|\mathbf{y}-\mathbf{W r}\|_{2}^{2}+\mu\|\mathbf{r}\|_{p}^{p} .
$$

When $p=1$, Eq. (2.12) is equivalent to the conventional reflectivity inversion method. In this paper, we will focus on the case where $p$ is greater than 0 and less than 1 , namely $L_{p}(0<p<1)$ regularization. The $L_{p}(0<p<1)$ regularization can recover sparser solutions from few measurements than the $L_{1}$ regularization [24]. It has been widely used 


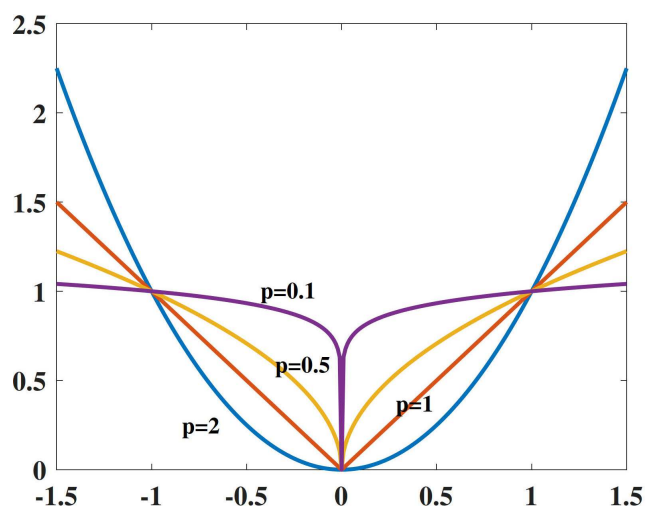

(a)

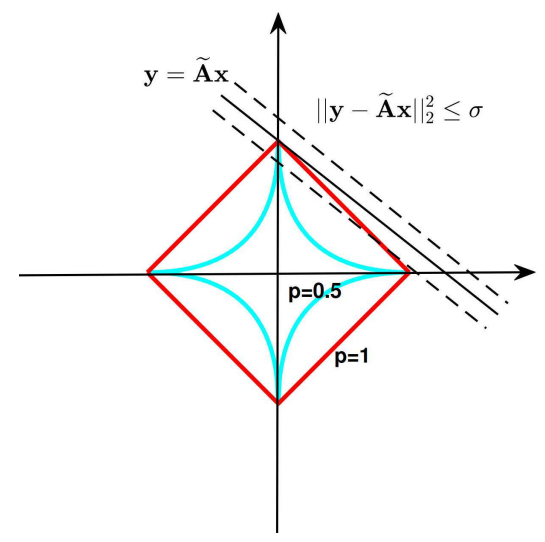

(b)

Figure 5: Diagram of $L_{p}$ norm. (a) the behavior of $|x|^{p}$ for various $p$-values, and (b) the feasible region of $L_{p}$ norm.

for compressive sensing and signal processing $[37,38]$, which indicates that the $L_{p}$ regularization is a potentially powerful new approach to the sparsity problem. Besides, the $L_{p}(0<p<1)$ regularization enjoys nice theoretical guarantee, which needs less restrictive isometry conditions than those needed for $L_{1}$ regularization [37].

Fig. 5(a) shows the curves of $|x|^{p}$ for various $p$-value, where the $|x|^{p}$ is the core of the norm computation. It shows that as $p$ goes to zero, the curve becomes an indicator function, which means that the behavior of $L_{p}(p \rightarrow 0)$ norm is closer to that of $L_{0}$ norm. Therefore the $L_{p}(0<p<1)$ norm constraint is more effective to promote sparsity than the $L_{1}$ norm constraint. In addition, the curve of $|x|^{p}$ norm becomes concave as the $p$-value goes to small, which implies that the $L_{p}(0<p<1)$ regularization improves the shortcoming of $L_{1}$ norm that is sensitive to the large value. Therefore the $L_{p}$ regularization has a better amplitude-preservation over $L_{1}$ regularization. Fig. 5(b) demonstrates the feasible region of $L_{p}(0<p \leq 1)$ norm. Obviously, if the data is disturbed by Gaussian noise, the solution of $L_{p}(0<p<1)$ regularization is sparser than that of the $L_{1}$ regularization since the dotted line intersects the contour line of the $L_{p}(0<p<1)$ norm near the axes. It means that the $L_{p}(0<p<1)$ regularization is robust to noise.

The performance of $L_{p}$ regularization depends on the selection of $p$-value. One may ask what the best $p$-value is. Empirical evidence suggests that $p=1 / 2$ is a good choice in at least a broad range of circumstances [39,40]. For example, the research of [22] reveals that the $L_{1 / 2}$ regularization is the sparsest and most robust when $1 / 2 \leq p<1$, and the $L_{p}$ regularizations have a similar performance to the $L_{1 / 2}$ regularization when $0<p<1 / 2$. In our study, many experiments have been tested and the conclusion is similar to that of [22]. Consequently, the $L_{1 / 2}$ regularization is chosen as a representative of the $L_{p}(0<p<1)$ regularization in this paper.

When $p$ is less than 1, Eq. (2.12) is generally difficult to be solved because of its nonconvex property. To address this issue, the HPP algorithm proposed by [29] uses the 


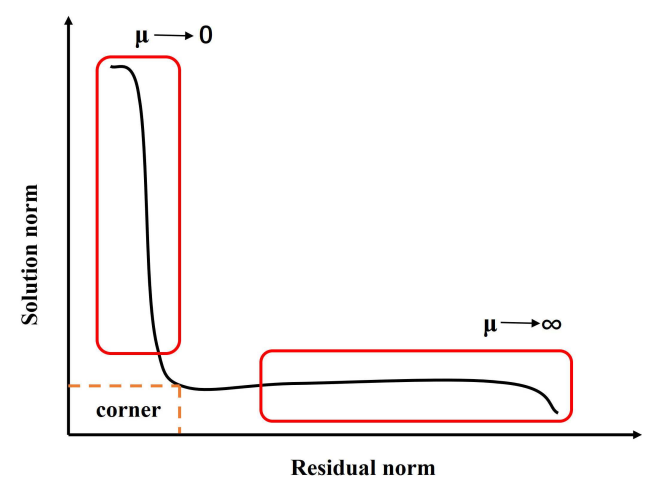

Figure 6: Diagram of L-curve.

Hadmard product parametrization to transform the $L_{p}(0<p<1)$ regularization into a series of simple $L_{2}$ regularizations, to which the existing $L_{2}$ regularization algorithms can be efficiently applied, so that the non-convex problem will be solved easily. Here, the SVD algorithm is applied to solve each Tikhonov regularization for high inversion accuracy, and the regularization parameter is determined adaptively by the L-curve method (Fig. 6). Therefore, the improved algorithm is called SVD-HPP algorithm. Appendix B shows the derivation of the SVD-HPP algorithm.

The pseudo-code of the proposed method is shown in Algorithm 2.

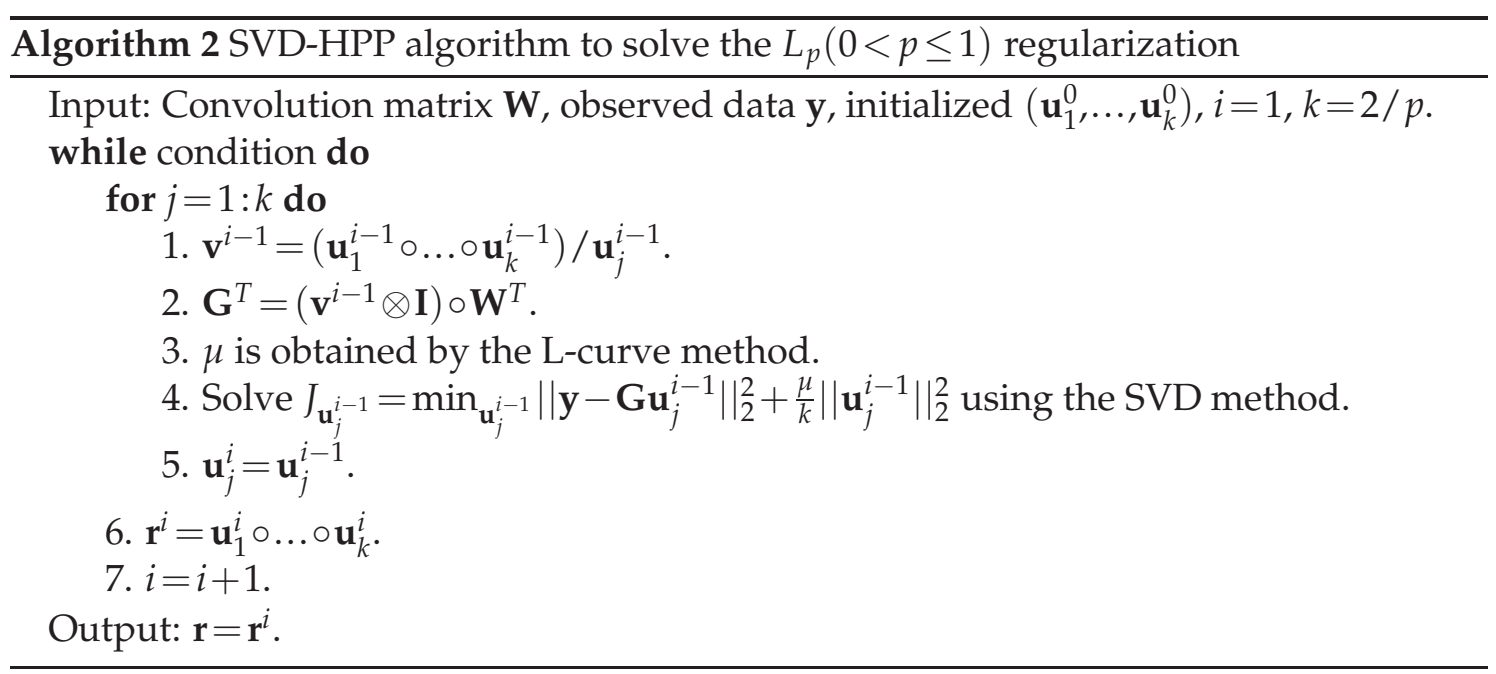

\subsection{Alternating iterative super-resolution inversion}

To solve the objective function (2.12), the first thing is to estimate an accurate seismic wavelet. Unfortunately, the estimation of seismic wavelet is full of challenges. Even 
though one obtains a seismic wavelet, the wavelet can not be guaranteed to be accurate [7], so that the estimated reflectivity may be doubtful. To achieve a credible reflectivity, the alternating iterative super-resolution inversion (AISRI) is an effective method for estimating the reflectivity and wavelet simultaneously, which avoids the effect of the wavelet estimation error.

Based on the commutative law of convolution, the following relation is derived,

$$
\mathbf{y}=\mathbf{W r}+\mathbf{n}=\mathbf{R} \mathbf{w}+\mathbf{n},
$$

where $\mathbf{R}$ denotes the convolution reflectivity matrix, and $\mathbf{w}$ refers to the seismic wavelet. The reflectivity can be estimated by solving the following cost function alternately,

$$
J_{\mathbf{r}, \mathbf{w}}=\min _{\mathbf{r}, \mathbf{w}} \frac{1}{2}\|\mathbf{y}-\mathbf{W r}\|_{2}^{2}+\Phi(\mathbf{r})+\Psi(\mathbf{w}),
$$

where $\Phi$ and $\Psi$ denote the constraint functions corresponding to $\mathbf{r}$ and $\mathbf{w}$, respectively. By setting the constraint functions reasonably, one can estimate credible reflectivity coefficients and seismic wavelet simultaneously. For the reflectivity, the $L_{p}(0<p<1)$ norm is used to constrain the reflectivity for pursuing a sparser solution, namely $\Phi(\mathbf{r})=\lambda_{1}|| \mathbf{r}||_{p}^{p}$ with regularization parameter $\lambda_{1}$. For the seismic wavelet, a mixed norm is designed to constrain the seismic wavelet, which has the following form

$$
\Psi(\mathbf{w})=\lambda_{2}|| \mathbf{w}\left\|_{\tilde{p}}^{\widetilde{p}}+\lambda_{3}\right\| \mathbf{w} \|_{2}^{2}
$$

where $0<\widetilde{p} \leq 1, \lambda_{2}$ and $\lambda_{3}$ denote the regularization parameters. The first $L_{\widetilde{p}}$ norm is added to impose a sparse constraint on the seismic wavelet, because the size of the seismic wavelet can be increased to $q$ by zero-filling. Here, it's enough to set $\widetilde{p}$ to 1 . The second $L_{2}$ norm is used to impose a smooth constraint on the seismic wavelet because of its smooth waveform at the non-zero position. Bringing the above two constraint functions into Eq. (2.14), the complete objective function is expressed as

$$
J_{\mathbf{r}, \mathbf{w}}=\min _{\mathbf{r}, \mathbf{w}} \frac{1}{2}\|\mathbf{y}-\mathbf{W r}\|_{2}^{2}+\lambda_{1}\|\mathbf{r}\|_{p}^{p}+\lambda_{2}\|\mathbf{w}\|_{\widetilde{p}}^{\widetilde{p}}+\lambda_{3}\|\mathbf{w}\|_{2}^{2}
$$

It is obvious that Eq. (2.16) is non-convex. Consequently, to solve this non-convex problem, we split the problem into two sub-problems solved in an iterative fashion. Considering multi-trace data, the objective functions of sub-problems are expressed as

$$
\begin{aligned}
& J_{\widetilde{\mathbf{R}}}=\min _{\widetilde{\mathbf{R}}} \frac{1}{2}\|\mathbf{Y}-\mathbf{W} \widetilde{\mathbf{R}}\|_{F}^{2}+\lambda_{1}\|\widetilde{\mathbf{R}}\|_{p}^{p}, \\
& J_{\mathbf{w}}=\min _{\mathbf{w}} \frac{1}{2}\left\|\operatorname{vec}(\mathbf{Y})-\overline{\mathbf{R}}_{k} \mathbf{w}\right\|_{2}^{2}+\lambda_{2}\|\mathbf{w}\|_{\widetilde{p}}^{\widetilde{p}}+\lambda_{3}\|\mathbf{w}\|_{2}^{2},
\end{aligned}
$$

where $\mathbf{Y}, \widetilde{\mathbf{R}}$, and $\mathbf{N}$ indicate multi-trace seismic data, reflectivity, and noise, respectively. The $k$-trace matrix $\overline{\mathbf{R}}_{k}$ can be given by

$$
\overline{\mathbf{R}}_{k}=\left[\mathbf{R}_{1} ; \mathbf{R}_{2} ; \cdots ; \mathbf{R}_{k}\right],
$$


where $\mathbf{R}_{i}$ denotes the convolution matrix consisting of the $i_{t h}$-trace reflectivity coefficient $\left(\widetilde{\mathbf{R}}_{i}\right)$. In Eq. (2.17) and Eq. (2.18), $\lambda_{1}, \lambda_{2}$, and $\lambda_{3}$ denote the constraint parameters that can be determined by experience. Because of the coupling of wavelet, the L-curve method can not be used to automatically determine the optimal regularization parameters, but it can provide a nontrivial range of potentially optimal parameter. Note that Eq. (2.17) and Eq. (2.18) hold under the assumption that there is only a seismic wavelet for the inverted multi-trace seismic data.

The pseudo-code of the AISRI method is shown in Algorithm 3.

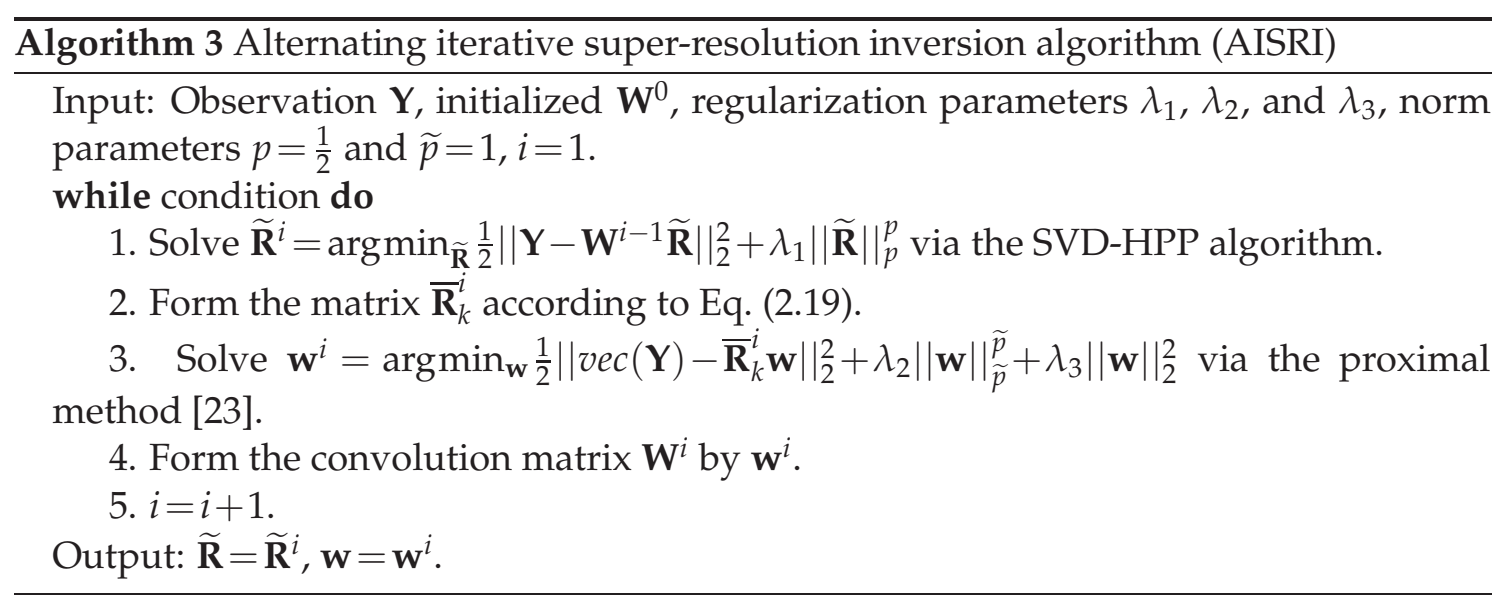

\section{Application}

In the following, we test the synthetic data to validate the proposed super-resolution inversion method and the proposed work-flow. Then, the proposed work-flow is applied to field data.

\subsection{Synthetic data examples}

1) The first experiment: In the first experiment, we have tested the super-resolution inversion method based on the $L_{p}(0<p<1)$ regularization, where the seismic wavelet is known. The noise-free synthetic seismic trace is firstly generated by substituting the reflectivity model and a $40 \mathrm{~Hz}$ Ricker wavelet with $30^{\circ}$ rotated phase into Eq. (2.10), as shown in Fig. 7. Then, the $L_{1 / 5}, L_{1 / 4}, L_{1 / 3}, L_{1 / 2}$, and $L_{1}$ regularizations are used for the reflectivity inversion, and the inversion results are shown in Fig. 7. Through observation, the inversion results are almost the same and very similar to the true reflectivity, which is indistinguishable from the naked eye. To show the differences between the inversion results, the error results are obtained by subtracting the estimated reflectivity coefficients from the true ones, as shown in Fig. 8. It's obvious that the error of the inversion result obtained by the $L_{1}$ regularization is the largest, and the $L_{1 / 2}$ regularization generates the 


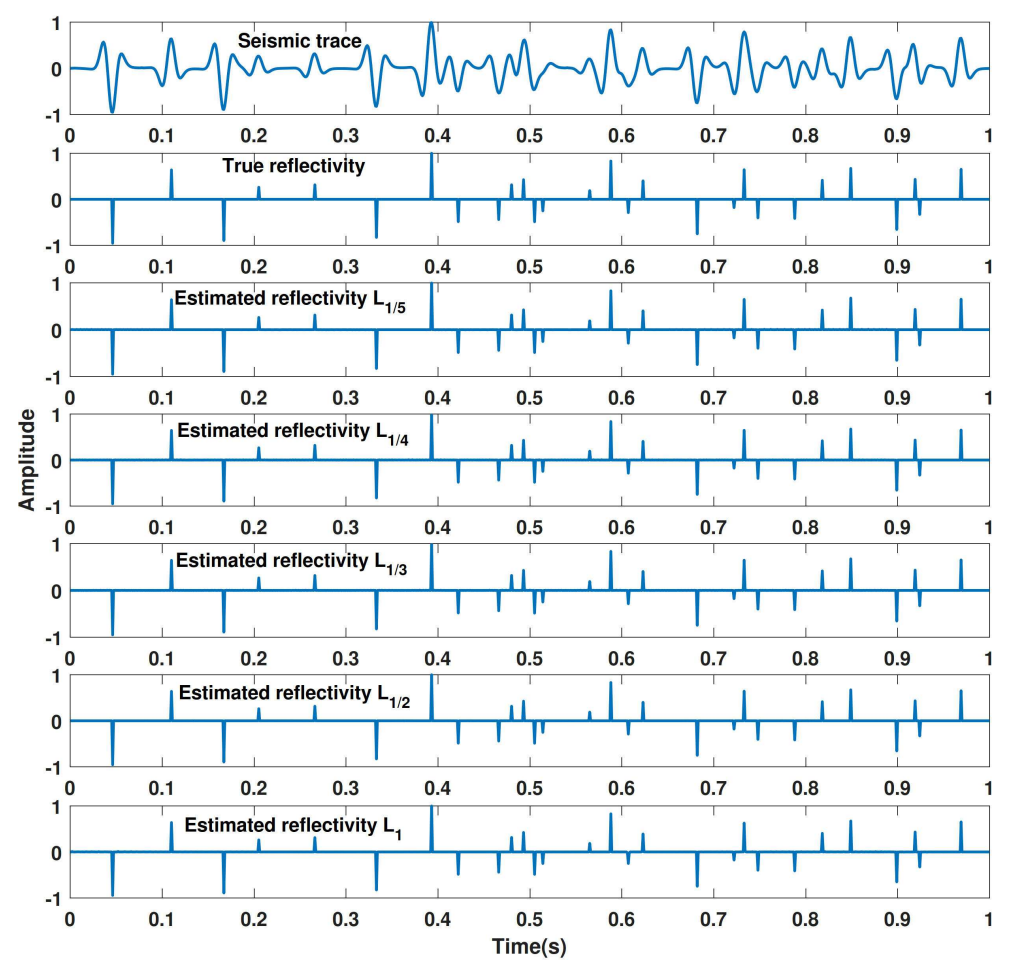

Figure 7: Reflectivity inversion based on the $L_{p}(0<p \leq 1)$ regularization in the noise-free case. The first line is the synthetic trace, the second line is the true reflectivity, and the other lines are the inversion results of the $L_{1 / 5}, L_{1 / 4}, L_{1 / 3}, L_{1 / 2}$, and $L_{1}$ regularizations, respectively.

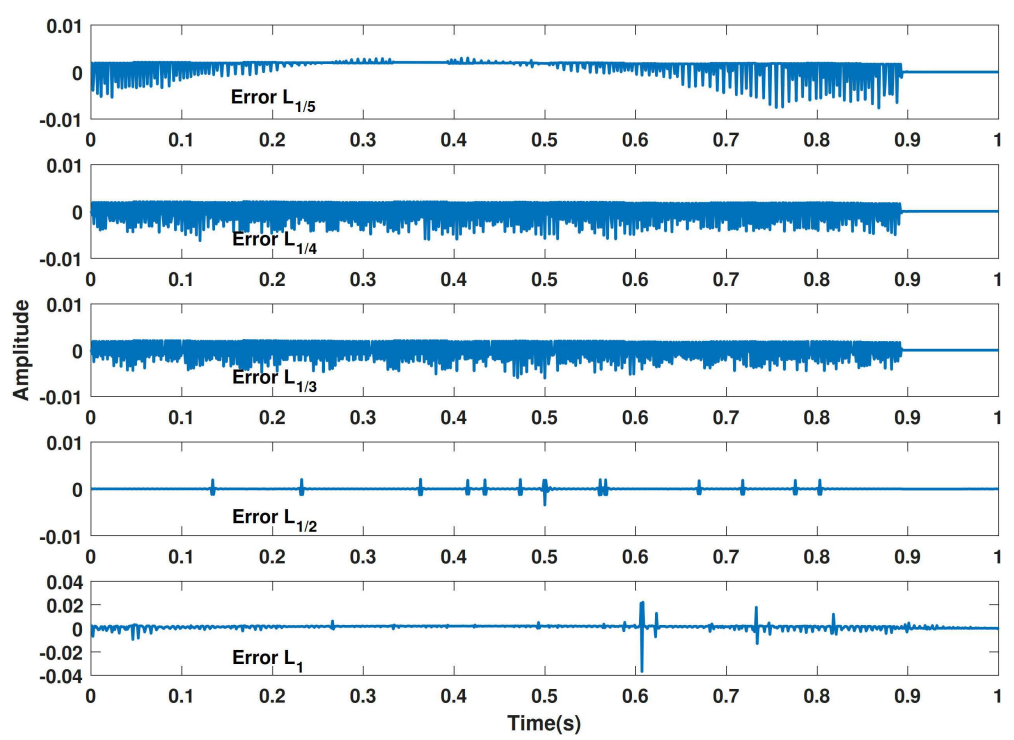

Figure 8: Error results corresponds to the inversion results in Fig. 7. 


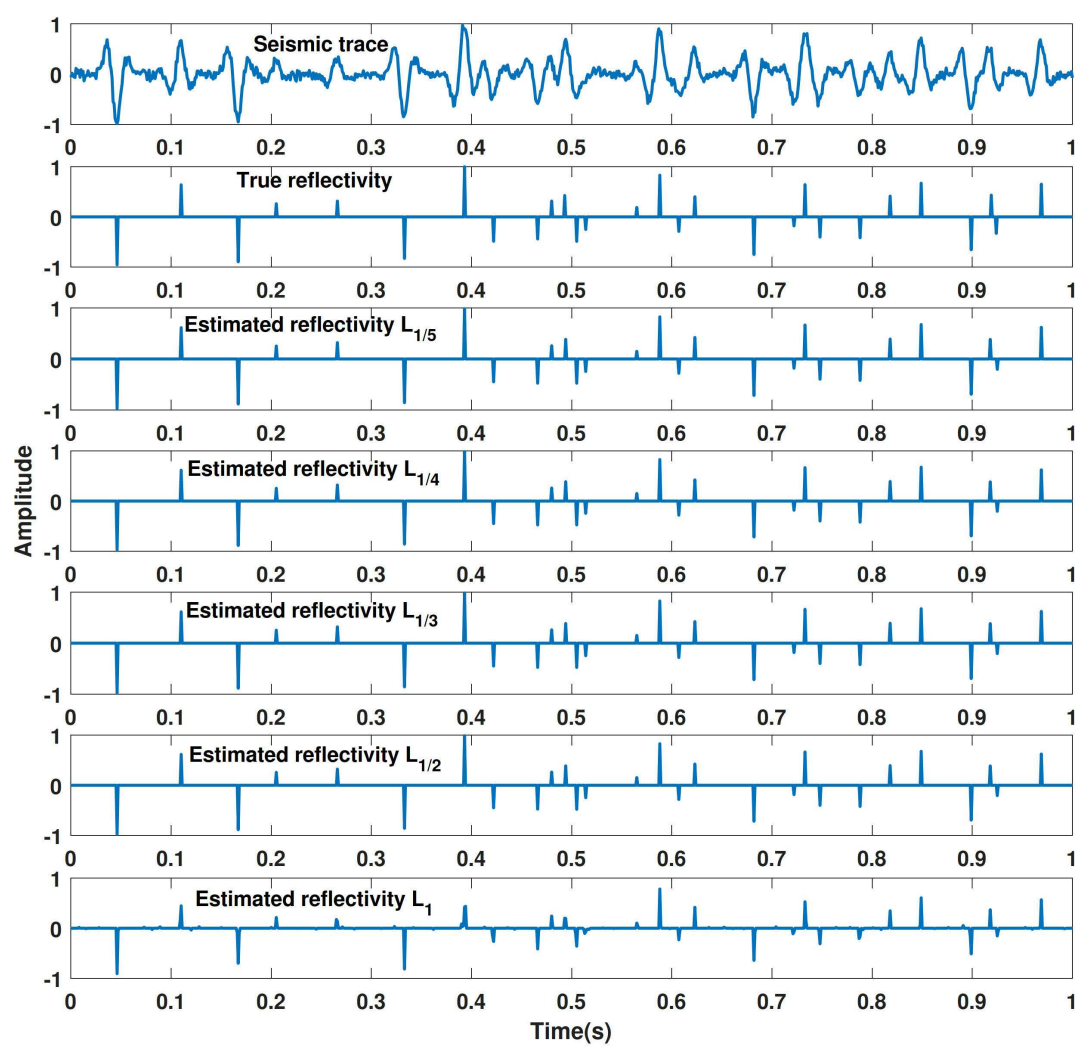

Figure 9: Reflectivity inversion based on the $L_{p}(0<p \leq 1)$ regularization in the case of noise. The first line is the noisy synthetic trace, the second line is the true reflectivity, and the other lines are the results of the $L_{1 / 5}$, $L_{1 / 4}, L_{1 / 3}, L_{1 / 2}$, and $L_{1}$ regularizations, respectively.

smallest error. Therefore, the $L_{p}(0<p<1)$ regularization can increase the inversion accuracy in contrast with the $L_{1}$ regularization, and the $L_{1 / 2}$ regularization performs better than the $L_{1 / 5}, L_{1 / 4}$, and $L_{1 / 3}$ regularizations in this experiment.

To test the stability of the super-resolution inversion method based on the $L_{p}(0<p<$ 1) regularization, the noise-free data shown in Fig. 7 is added $20 \%$ Gaussian noise, and the noisy data is shown in Fig. 9. Fig. 9 shows the inversion results estimated by the $L_{1 / 5}$, $L_{1 / 4}, L_{1 / 3}, L_{1 / 2}$, and $L_{1}$ regularizations. Obviously, the inversion results of the $L_{1 / 5}, L_{1 / 4}$, $L_{1 / 3}$, and $L_{1 / 2}$ regularizations with less energy loss are better than that of the $L_{1}$ regularization. Furthermore, Fig. 10 shows the error results corresponding to the $L_{1 / 5}, L_{1 / 4}$, $L_{1 / 3}$, and $L_{1 / 2}$ regularizations. They are similar and smaller than the error corresponding to the $L_{1}$ regularization, which demonstrates that the $L_{p}(0<p<1)$ regularization is more robust to random noise. Comparatively, there is a significant energy loss and some false spikes in the result of the $L_{1}$ regularization with large error, as shown in Fig. 9 and Fig. 10. Based on the above tests, the $L_{1 / 2}$ regularization can be selected as a representative of the $L_{p}(0<p<1)$ regularization to invert a credible reflectivity. 


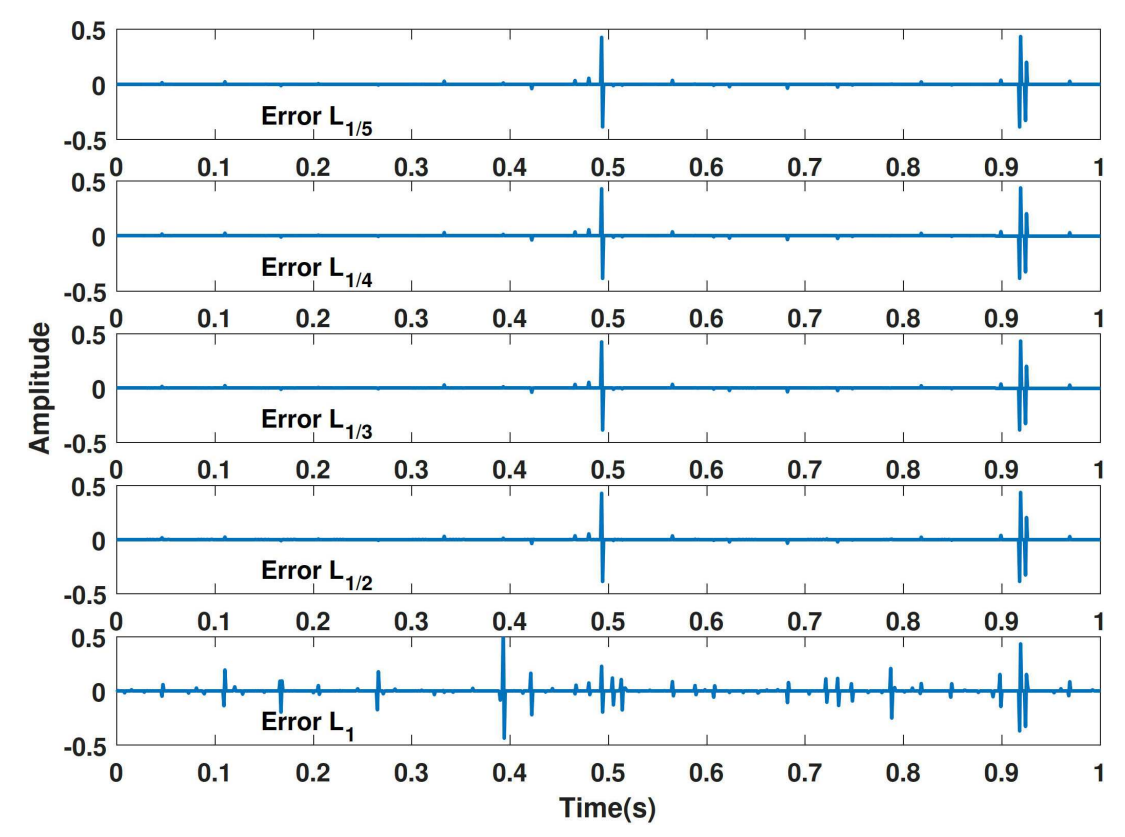

Figure 10: Error results corresponds to the inversion results in Fig. 9.

In the first experiment, the super-resolution inversion based on the $L_{p}(0<p<1)$ regularization is solved by the SVD-HPP algorithm, and the regularization parameter is determined by the L-curve method at each iteration. Fig. 11(a) and Fig. 11(b) show the determined regularization parameters of the above tests. It can be seen that the $L_{p}(0<p<1)$ regularization has faster convergence and requires fewer iterations than the $L_{1}$ regularization under the same stopping conditions. In addition, the parameter of the $L_{p}(0<p<1)$ regularization is smaller than that of the $L_{1}$ regularization during convergence, which indicates that the former has a stronger sparse constraint, and the parameter increases in the presence of noise to reduce false signals.

2) The second experiment: The first experiment is done base on a known seismic wavelet. Nevertheless, in reality, the seismic wavelet is unknown, and the error of the estimated wavelet will affect the estimation of reflectivity. The AISRI method is an effective strategy to mitigate the influence of the seismic wavelet, which is tested in this experiment. First, an initial wavelet is obtained by the method proposed by [33] before inversion. Then, the $L_{1 / 2}$ norm is used to constrain the reflectivity as a representative of the $L_{p}(0<p<1)$ norm. Fig. 12 shows the inversion results obtained by AISRI under the condition of noisefree, where the synthetic seismic trace is the same as that in Fig. 7. Through observation, one can learn that the $L_{1 / 2}$ regularization performs better than the $L_{1}$ regularization in the case of the same wavelet constraint function. In other word, the $L_{1 / 2}$ regularization can obtain a relatively amplitude-preserving reflectivity in contrast with the $L_{1}$ regularization. The corresponding estimated wavelets are similar and close to the true wavelet. 


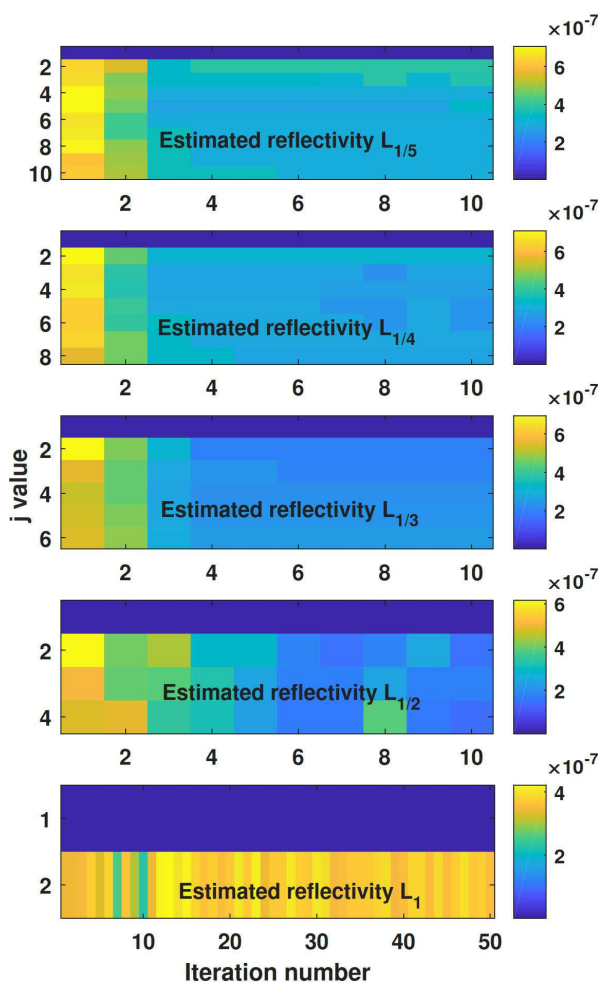

(a)
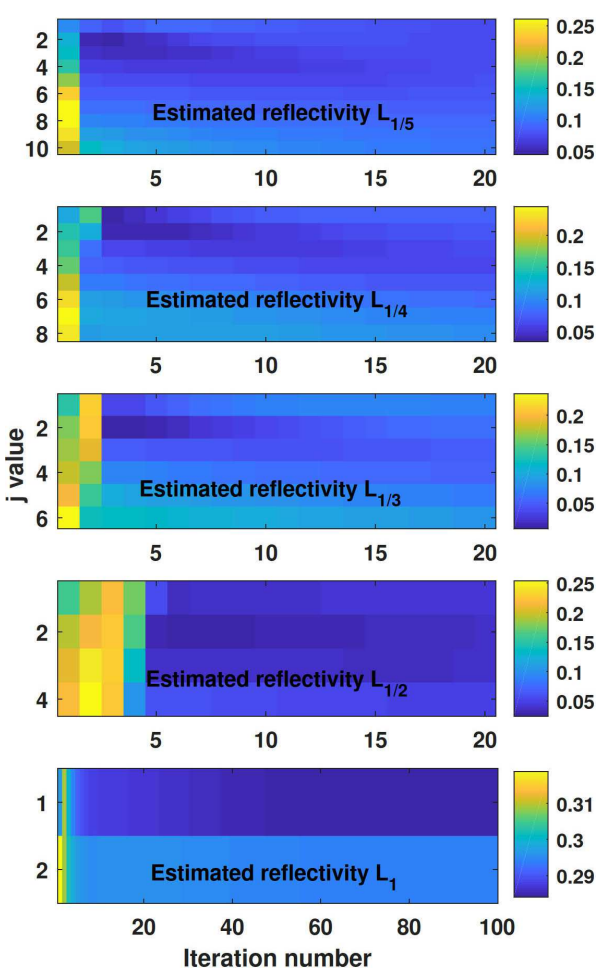

(b)

Figure 11: Determination of the regularization parameters. (a) the regularization parameters of the $L_{1 / 5}, L_{1 / 4}$, $L_{1 / 3}, L_{1 / 2}$, and $L_{1}$ regularizations in the noise-free case, and (b) the regularization parameters of the $L_{1 / 5}$, $L_{1 / 4}, L_{1 / 3}, L_{1 / 2}$, and $L_{1}$ regularizations in the case of noise.

Fig. 13 displays the inversion results of noisy data (shown in Fig. 9). The reflectivity inverted by the AISRI method with the $L_{1 / 2}$ regularization is recovered well, where the energy relationship is preserved and there are few false spikes. Even though there are some differences between the estimated and true wavelets, it seems to have little effect on the inversion reflectivity. By comparison, there is an obvious energy loss especially for some weak signals in the inversion results of the AISRI method with the $L_{1}$ regularization, but the inversion wavelet is better than that of the AISRI method with the $L_{1 / 2}$ regularization, the influence of which is not apparent. Finally and interestingly, we investigate the inversion results of the first and second experiments and find that the estimated reflectivity in the second experiment is inferior to that in the first experiment due to the couplings of wavelet and reflectivity.

3) The third experiment: In the third experiment, the proposed work-flow is tested. The non-stationary seismic trace shown in Fig. 14 is generated by applying the attenuation function to the synthetic data in Fig. 7 [41], where $Q=80$ for $t=(0-0.55)$ s and $Q=100$ for $t=(0.55-1) \mathrm{s}$. The true reflectivity model is the same as that in Fig. 7. Firstly, the 


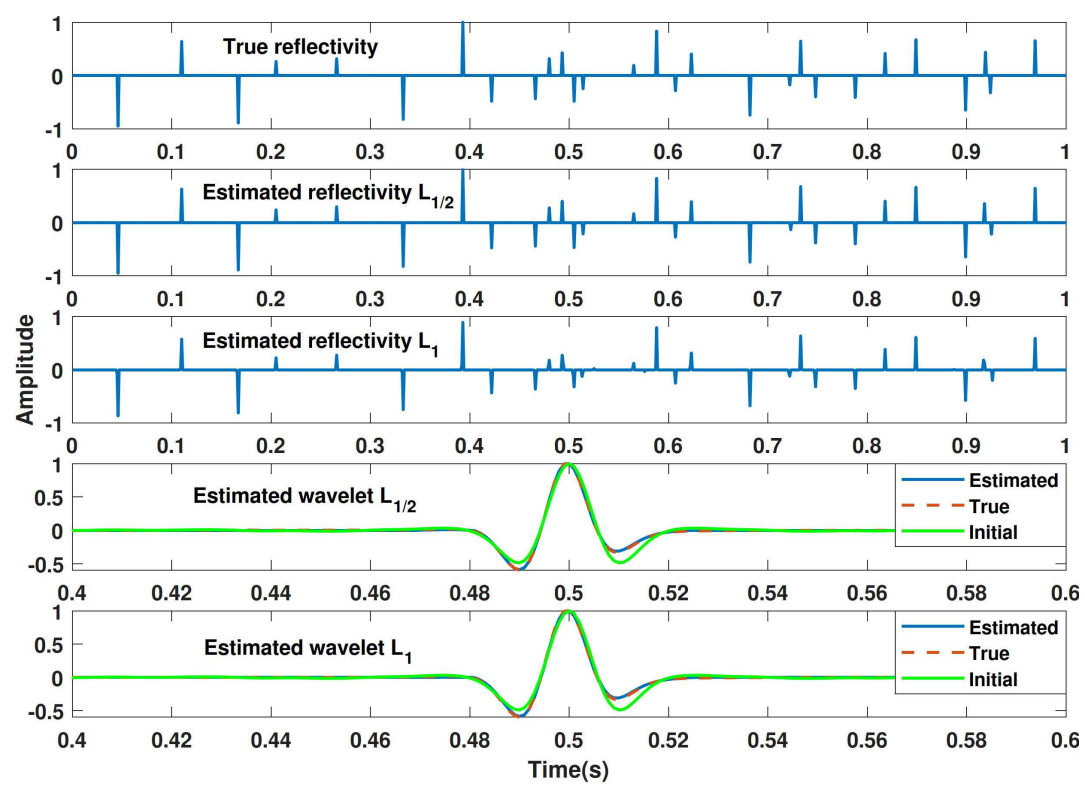

Figure 12: Test of the AISRI method in the noise-free case. The first line is the true reflectivity, the second line is the estimated reflectivity by the AISRI method with the $L_{1 / 2}$ regularization, the third line is the estimated reflectivity using the AISRI method with the $L_{1}$ regularization, and the other lines are the corresponding estimated seismic wavelets.

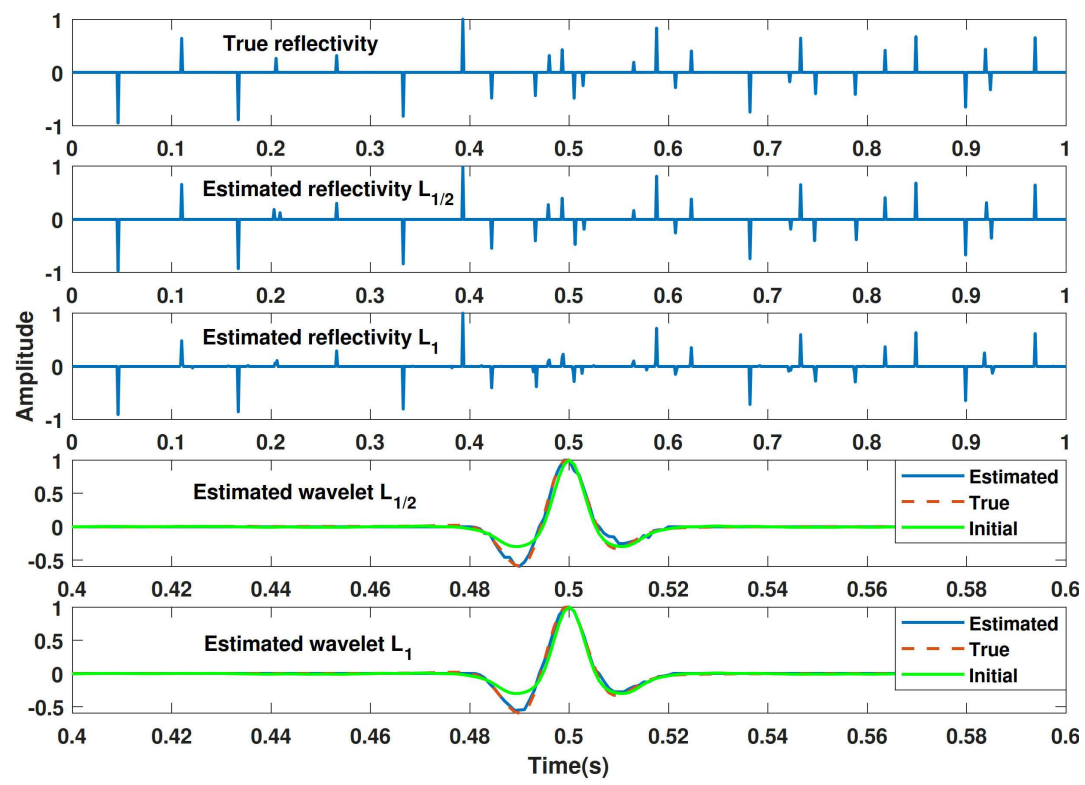

Figure 13: Test of the AISRI method in the case of noise. The first line is the true reflectivity, the second line is the estimated reflectivity by the AISRI method with the $L_{1 / 2}$ regularization, the third line is the estimated reflectivity using the AISRI method with the $L_{1}$ regularization, and the other lines are the corresponding estimated seismic wavelets. 


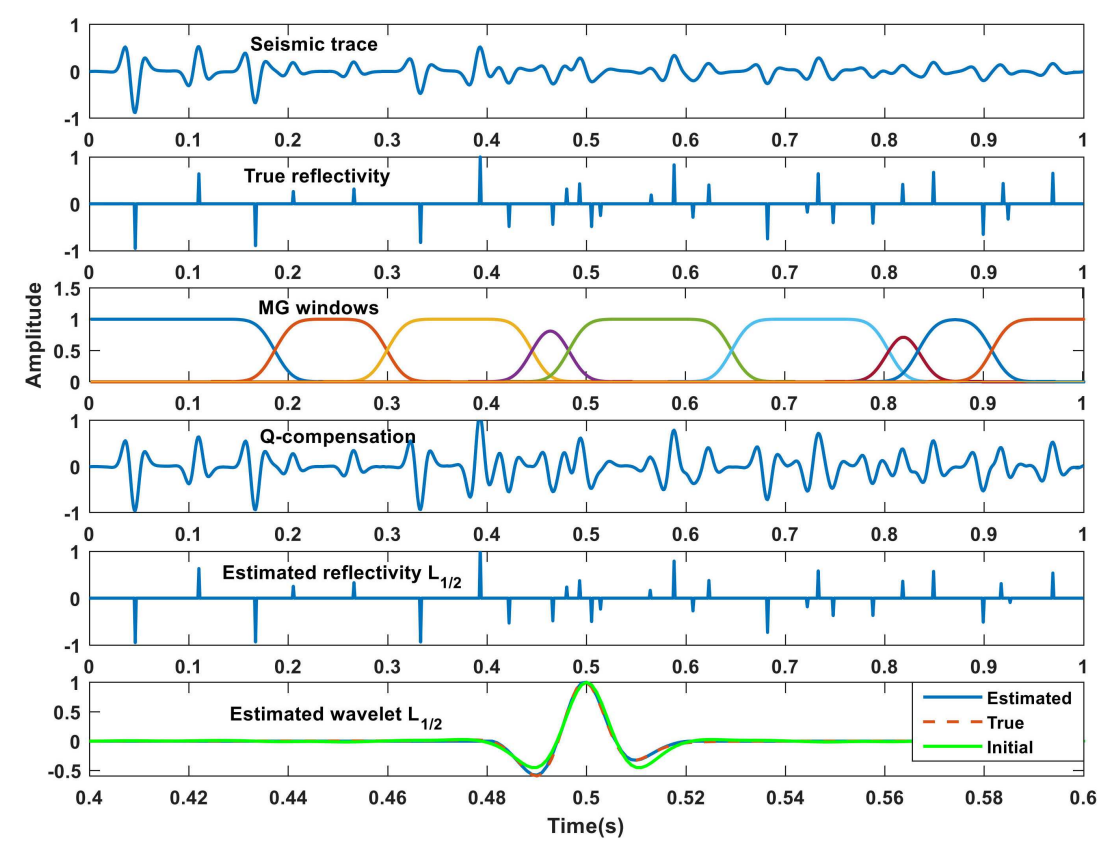

Figure 14: Test of the proposed work-flow in the noise-free case. The first line is the synthetic non-stationary data $(Q=80$ for $t=(0-0.55) \mathrm{s}$ and $Q=100$ for $t=(0.55-1) \mathrm{s})$, the second line is the true reflectivity, the third line is the final MG windows, the fourth line is the compensation result, the fifth line is the estimated reflectivity, and the last line is the estimated wavelet.

MG windows are constructed according to the theory of adaptive partition. Then, the equivalent $Q$ values are estimated in the MG windows. Note that the estimated $Q$ values are not displayed here, because the accuracy of the estimated $Q$ values will be reflected in the following compensation results. The $Q$-compensation result obtained by ACSD is shown in Fig. 14. Through observation on the compensation result and the synthetic stationary trace shown in Fig. 7, the compensation result is similar to the stationary data and doesn't magnify the numerical error. It implies that the ACSD method can compensate for the non-stationary seismic data stably and the estimated $Q$-value is close to the true $Q$-value. To recover the reflectivity, the AISRI method is used to invert the compensation data. The inversion results are shown in Fig. 14. It can be observed that the inverted reflectivity approaches the true reflectivity and the inverted wavelet approaches the true wavelet.

To further validate the effectiveness of the proposed work-flow, we add $10 \%$ random noise to the non-stationary trace, as shown in Fig. 15. Because of the existence of noise, the $\mathrm{MG}$ windows are different from those in the noise-free case. The main reason is that the noisy data need to be smoothed when estimating the envelope of seismic data, and thus the initial MG windows are changed. Based on these MG windows, the $Q$-value is estimated. Then the ACSD method is implemented to compensate for the non-stationary data, and the compensation result is displayed in Fig. 15. It can be seen that the ampli- 


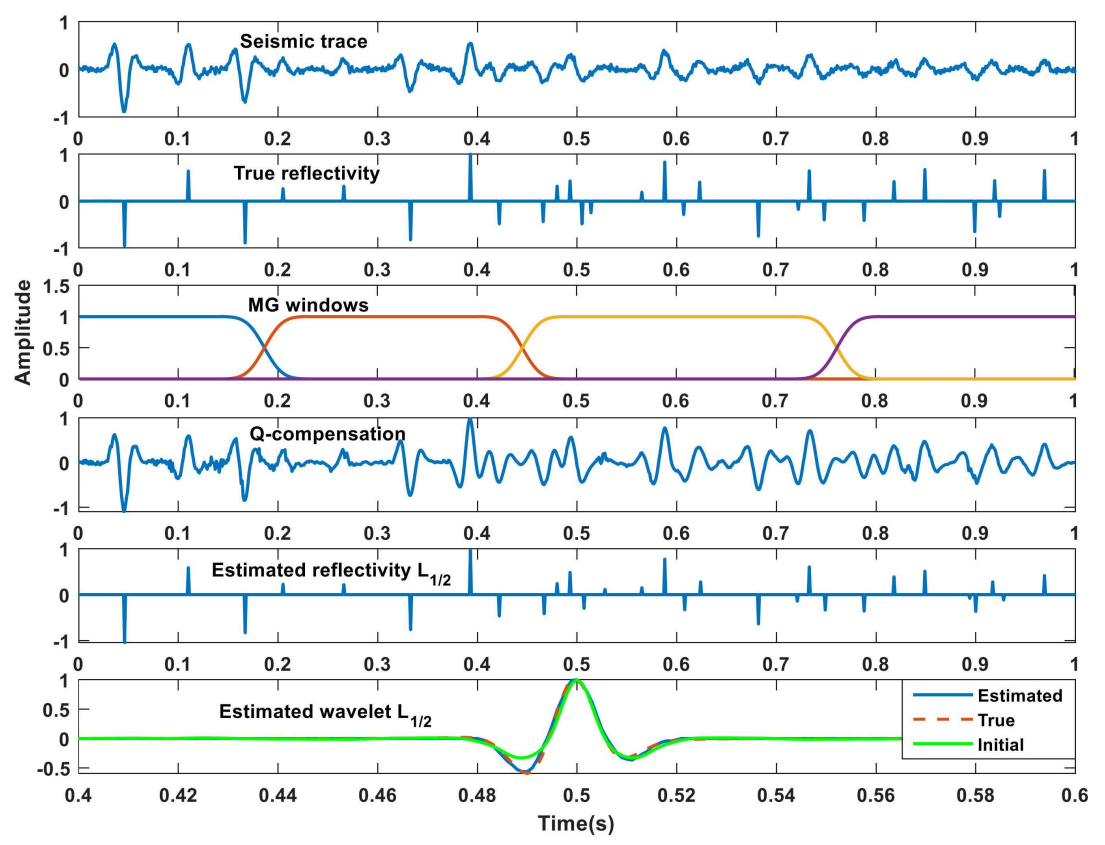

Figure 15: Test of the proposed work-flow in the case of noise. The first line is the synthetic non-stationary data $(Q=80$ for $t=(0-0.55) \mathrm{s}$ and $Q=100$ for $t=(0.55-1) \mathrm{s})$ with $10 \%$ random noise, the second line is the true reflectivity, the third line is the final MG windows, the fourth line is the compensation result, the fifth line is the estimated reflectivity, and the last line is the estimated wavelet.

tude of the attenuated result is approximately recovered and the noise is not amplified obviously, only very little noise amplification. After compensation, the reflectivity is estimated by the AISRI method, as shown in Fig. 15. The estimated reflectivity demonstrates that most spike signals are inverted well with few signals loss, and the estimated wavelet is close to the true wavelet which implies that the wavelet inversion is more robust to noise.

To test the lateral continuity of the inversion results using the proposed work-flow, a $2 \mathrm{D}$ data is tested. Note that the inversion method is implemented in a single-trace manner to facilitate the computer processing and reduce the block processing in this paper. Fig. 16(a) shows the true reflectivity model which is a portion of the Marmousi II model. Fig. 16(b) shows the synthetic noise-free data generated by substituting the reflectivity model, a $40 \mathrm{~Hz}$ Ricker wavelet with $30^{\circ}$ phase, and $Q$ values (i.e., $Q=80$ for $t=(0-0.8) \mathrm{s}$ and $Q=100$ for $t=(0.8-1.56) \mathrm{s})$ into non-stationary equation [41]. Fig. 16(e) displays the noisy data, where the noise level is $5 \%$ of the normalized noise (the maximum is 1 ). Then the proposed work-flow is applied to process the noise-free and noisy data. The compensation results and inverted reflectivity coefficients are shown in Fig. 16. Through observation, the compensation results and the inverted reflectivity profiles have good lateral continuity and remain the energy relationship, especially in the case of noise-free. 


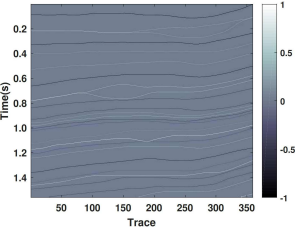

(a)

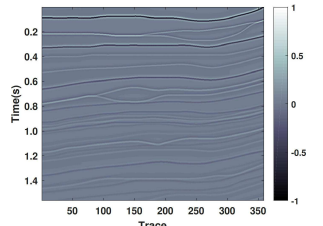

(b)

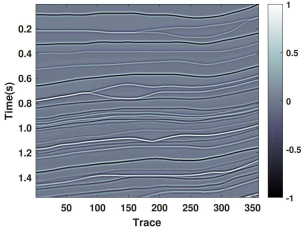

(c)

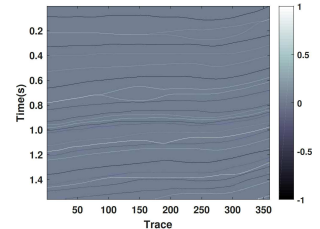

(d)

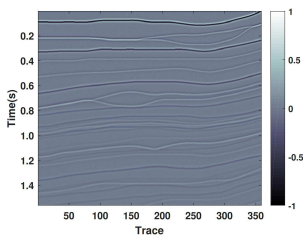

(e)

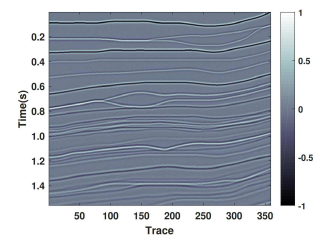

(f)

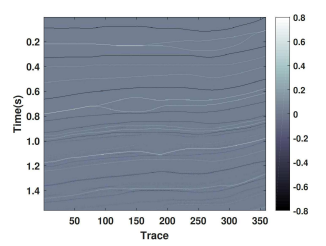

(g)

Figure 16: Test of the proposed work-flow for 2D synthetic data. (a) the true reflectivity model, (b) the synthetic non-stationary data $(Q=80$ for $t=(0-0.8) \mathrm{s}$ and $Q=100$ for $t=(0.8-1.56) \mathrm{s}),(\mathrm{c})$ the compensation result corresponding to Fig. 16(b), (d) the estimated reflectivity corresponding to Fig. 16(c), (e) the synthetic nonstationary data with $5 \%$ of the normalized noise (the maximum is 1 ), (f) the compensation result corresponding to Fig. 16(e), and (g) the estimated reflectivity corresponding to Fig. 16(f).

\subsection{Field data example}

In this section, we use a 2D field data to test the proposed work-flow. Fig. 17 shows a poststack field data, which is provided by China National Offshore Oil Corporation $(\mathrm{CNOOC})$. The size of this seismic data is $601 \times 701$ (time sampling number $\times$ trace number), and the time interval is $1 \mathrm{~ms}$. Besides, the field data has been processed via some conventional processing flows, including geometric spreading amplitude compensation, random noise attenuation, stack, and poststack time migration and so on.

The resolution of Fig. 17 is low because of the existing attenuation in the subsurface medium, especially in the deep part. To enhance the resolution of the field data, we use the proposed work-flow to compensate for the field data and then extract the reflectivity. Fig. 18 displays the compensation result by the ACSD method. After compensation, the amplitude is compensated, and the vertical resolution and lateral continuity are improved, especially at the location marked by black arrows and green ellipses, which is conducive to characterizing the geological structures by geologists. Note that Fig. 17 and Fig. 18 have the same color scale. Furthermore, Fig. 19 shows there normalized amplitude spectra, where the first is the average amplitude spectrum of the whole time section, the second is the average amplitude spectrum of the first half time section, and the third is the average amplitude spectrum of the second half time section. Comparing the amplitude spectra before and after compensation, the frequency band after compensation is obviously broadened. Therefore, the compensation result improves the inconsistency of the amplitudes in deep and shallow layers, and the seismic wavelet existed in the compensation result is nearly stationary in the propagation process. 


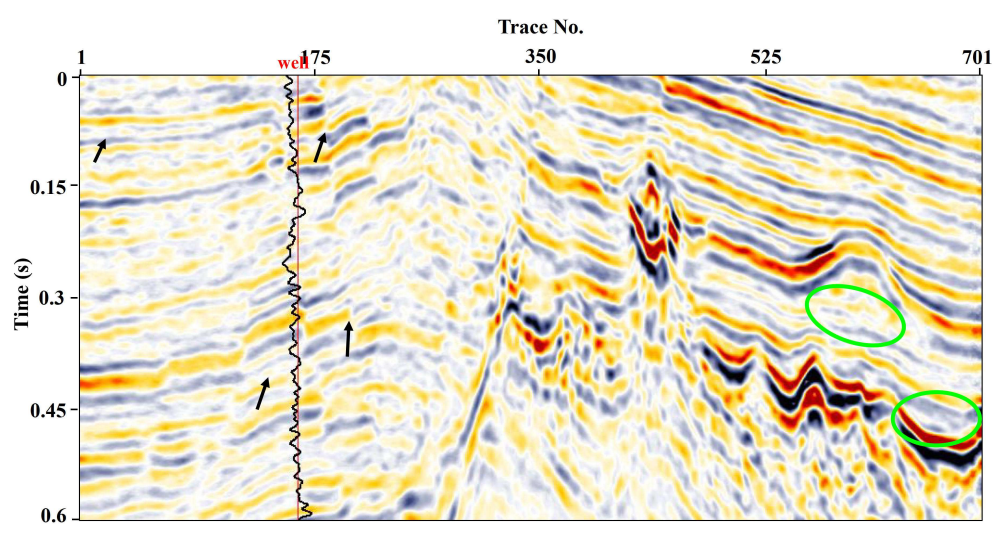

Figure 17: Field data.

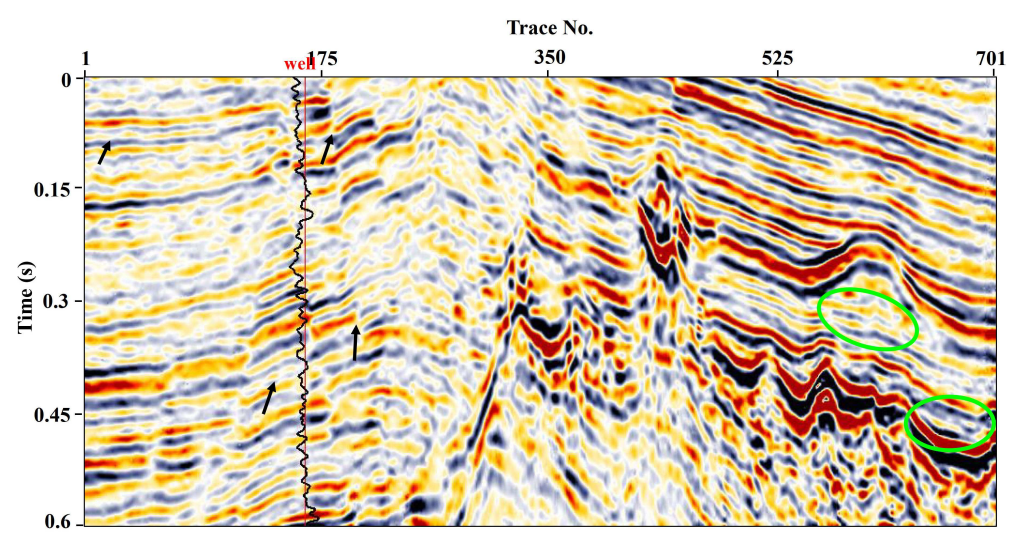

Figure 18: Compensation result using the ACSD method.

After compensation, the AISRI method is performed to recover the reflectivity from the compensation profile. Before inversion, an initial wavelet is obtained by the method proposed by [33], where the amplitude is the average amplitude of the whole time section. The inversion method is implemented in a single-trace manner. Fig. 20 shows the estimated wavelets of the first 20-trace data, each of which has a similar waveform. Fig. 21 shows the inverted reflectivity. It can be seen that the AISRI method can recover the reflectivity well, and the structure of field data is preserved. For instance, the curve structure is with good lateral continuity, and the details are described clearly, especially in the green ellipses and black arrow positions. Moreover, the energy relationship is consistent with that of the compensation data. To validate the accuracy of the inversion result, an acoustic impedance log is inserted in Fig. 17, Fig. 18, and Fig. 21, which is filtered by a low-pass filter with $250 \mathrm{~Hz}$ cutoff frequency. Through observation, the inversion results near the well can match the locations of different impedance layer boundaries well, 

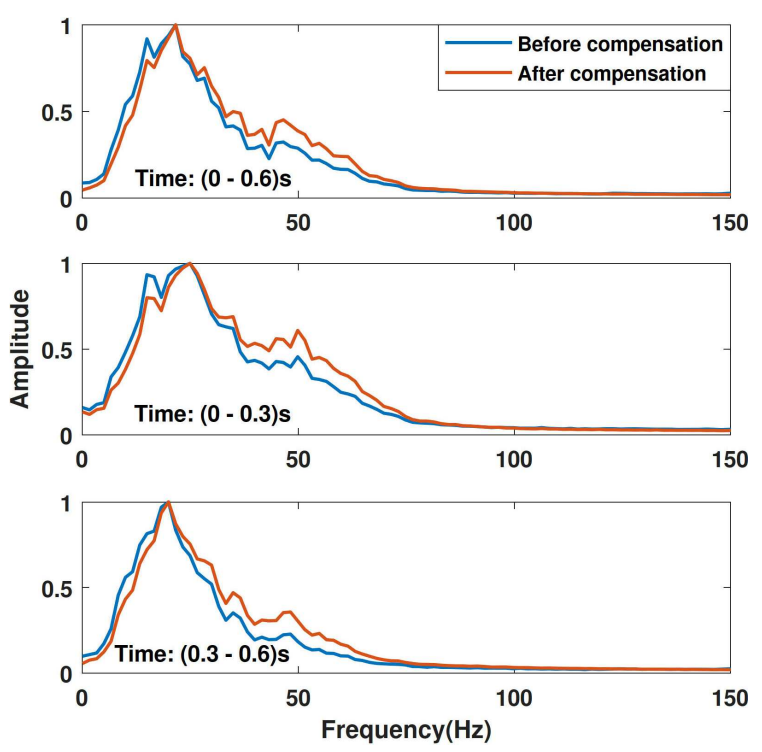

Figure 19: Normalized amplitude spectra. The first is the average amplitude spectrum of the whole time section, the second is the average amplitude spectrum of the first half time section, and the third is the average amplitude spectrum of the second half time section.

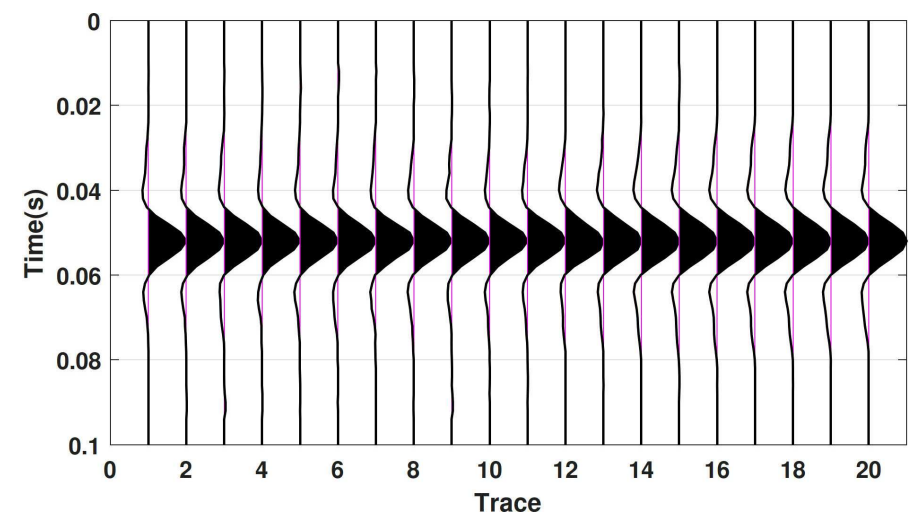

Figure 20: Estimated seismic wavelets of the first 20-trace data.

which illustrates the accuracy of the estimated reflectivity using the proposed method near the well. Fig. 22 shows the amplitude spectra of the compensation data and estimated reflectivity near the well. Obviously, the resolution of seismic data is significantly improved, and there is almost no low-frequency loss.

Finally, for clear observation on the results of each step in this work-flow, Fig. 23(a) and Fig. 23(b) show the estimated results of the $170_{t h}$ and $550_{\text {th }}$ traces of data using the proposed work-flow, respectively. Obviously, the compensated result is well without obvious false information and consistent with the original seismic data; the reflectivity is recovered reasonably. 


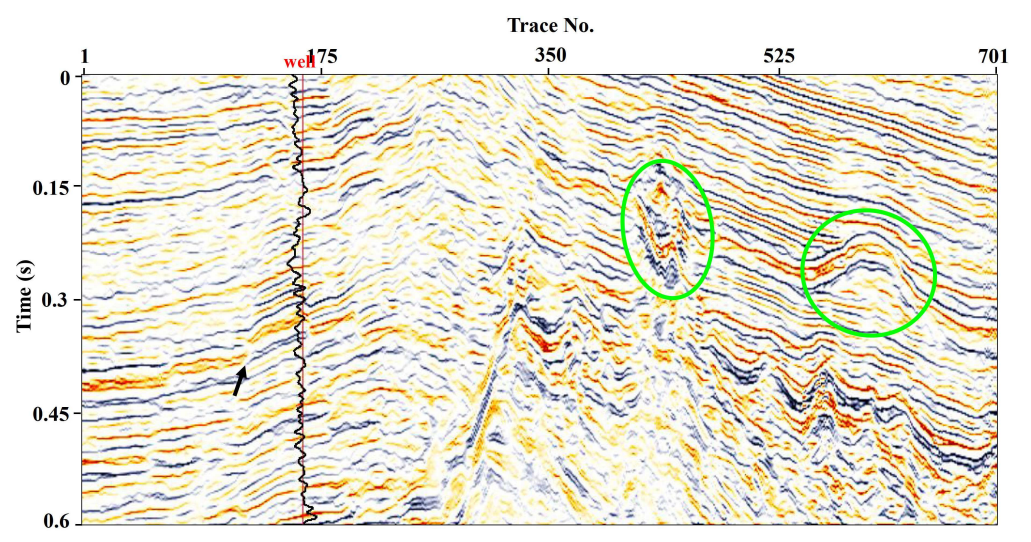

Figure 21: Estimated reflectivity using the AISRI method.

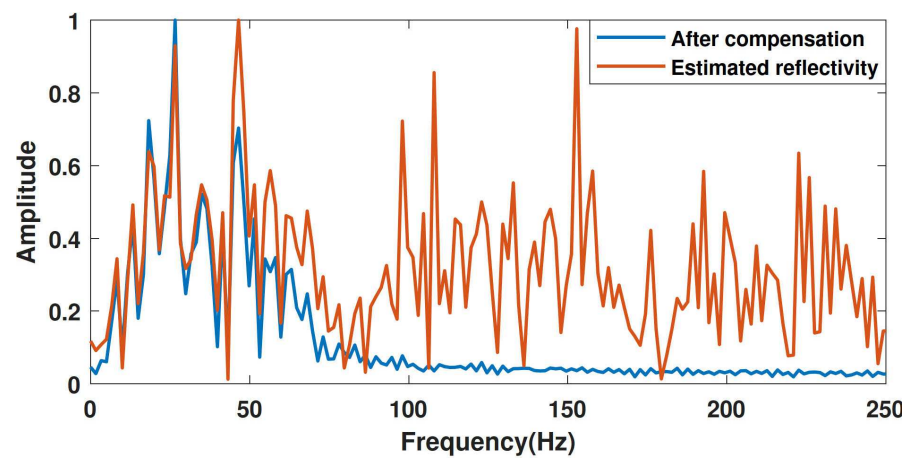

Figure 22: Amplitude spectrum of the estimated reflectivity near the well.

The above results show that the proposed work-flow can compensate for the field data and extract the reflectivity from the compensation data well.

\section{Discussion}

A relative amplitude-preserving super-resolution inversion method is proposed, which can be used to invert the non-stationary reflection seismic data. There are three main contributions in this paper. The first one is that an effective non-stationary inversion frame is devised to implement the super-resolution inversion of non-stationary seismic data, which mainly includes two-step, i.e., the stabilization of non-stationary seismic traces and the super-resolution inversion. The second one is that the $L_{p}(0<p<1)$ regularization is imposed to constrain the reflectivity, which can obtain a sparser solution. In addition, a simple and effective algorithm is provided to solve the $L_{p}(0<p \leq 1)$ regularization problem, and the regularization parameters at each iteration can be determined 


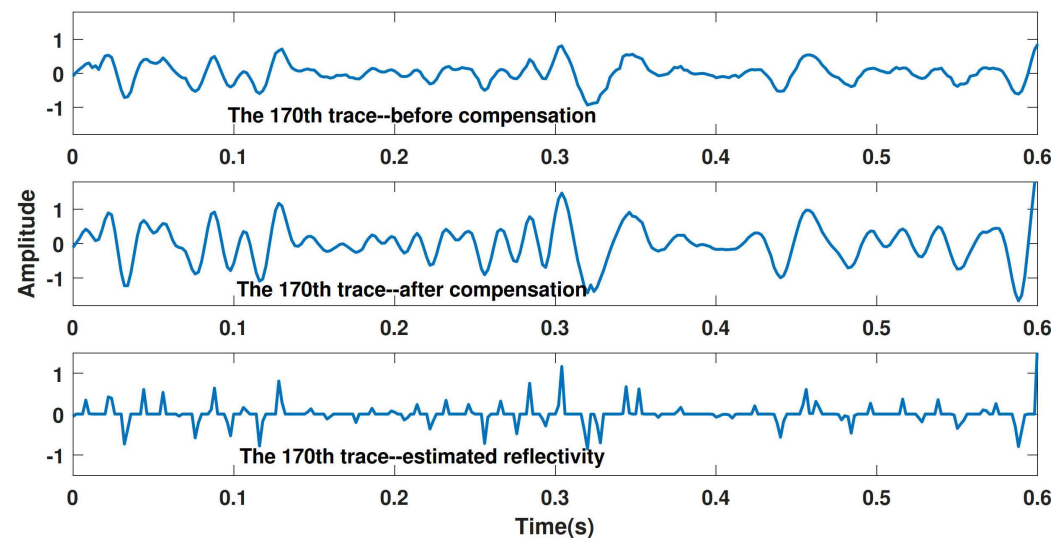

(a)
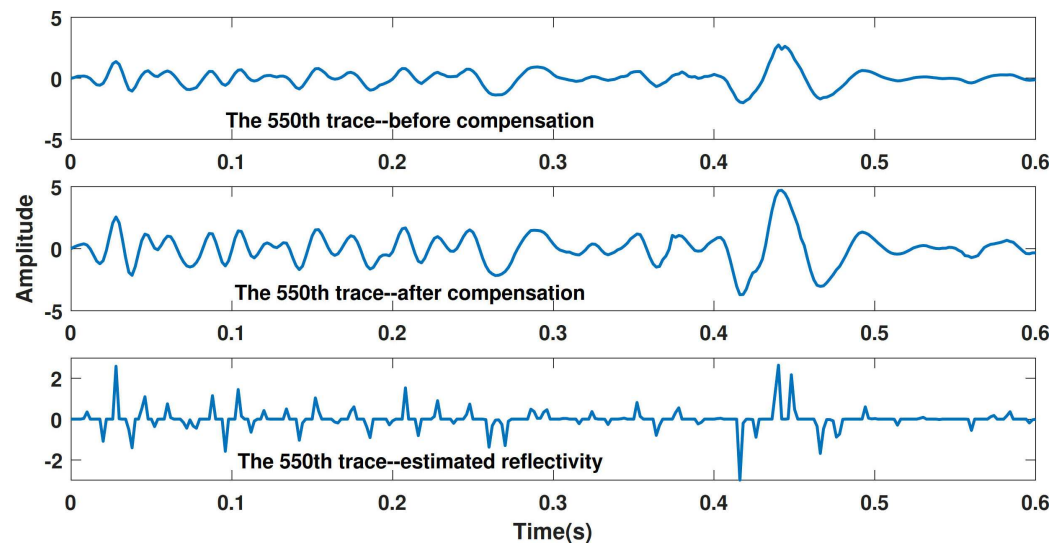

(b)

Figure 23: Test of proposed work-flow for field data. (a) the results of the $170_{t h}$ trace of data, and (b) the results of the $550_{t h}$ trace of data.

adaptively in solving the problem. The last one is that the AISRI method is introduced to estimate the reflectivity and seismic wavelet simultaneously in the case of an unknown wavelet, which can reduce the influence brought by an estimated wavelet.

However, it should be noted that there are some unresolved problems. In the stabilization process of non-stationary seismic traces, the accuracy of the estimated $Q$ value is affected by various factors, e.g., the influence of thin layer interference. To obtain credible $Q$ values, the well logs will be considered in our future work. The other problem is the determination of all regularization parameters for the AISRI. It is full of challenges to determine multiple parameters at the same time because of their couplings. Though some methods, such as GCV, have been proposed to determine multiple parameters, the computational cost is large and the methods are affected by noise. Recently, learning in a functional analytic regularization has been a hot topic [42], which opens up a new way to 
solve the inverse problem. In our future work, we will implement the super-resolution inversion using the deep learning methods, where the regularization parameters and constraint functions can be learned. In addition, the proposed method is suitable for a single trace or 2D data. However, we need to vectorize the $2 \mathrm{D}$ data into single column data to finish the inversion when considering $2 \mathrm{D}$ data. Therefore, the lateral information of 2D data is not fully used in the proposed method, which may affect the lateral continuity of inversion results. To obtain better results, the proposed method can be extended to implement the $L_{p, q}(0<p \leq 1, q \geq 1)$ regularization inversion [43] which adds the $L_{p}(0<p \leq 1)$ constraint along the time direction and the $L_{q}(q \geq 1)$ constraint along the space direction.

\section{Conclusion}

A non-stationary super-resolution inversion system is proposed in this paper, which can be applied in the non-stationary field data. There is mainly two-step, the first step of which devotes to making the non-stationary seismic data stationary and the second step of which is the super-resolution inversion of stationary seismic traces. In the first step, it includes the division of non-stationary seismic traces, the extraction of wavelet amplitude spectrum, the calculation of $Q$ values, and the compensation of non-stationary seismic traces. The above methods are based on the existing researches, which are developed and applied by us effectively. The super-resolution inversion in the second step is a novel inversion method proposed in this paper to obtain reflectivity, where the $L_{p}(0<p<1)$ norm and a mixed-norm are imposed to constrain the reflectivity and wavelet, respectively; Further, a novel SVD-HPP algorithm with adaptive regularization parameters is introduced to solve the $L_{p}(0<p \leq 1)$ regularization. Finally, the synthetic and field data examples validate the effectiveness of the proposed methods.

\section{Acknowledgments}

The authors greatly appreciate the research is supported by National Key R\&D Program of China grant No. 2018YFC0603501, and the National Science and Technology Major Projects under grants No. 2016ZX05024-001-007 and No. 2017ZX05069 for their financial supports. The authors also thank the Fundamental Research Funds for the Central Universities under grant No. xzy022020053, and the CNOOC Research Center for providing the data.

\section{Appendix A: Determination of parameters in the COM method}

There are three parameters, $c_{p}, \alpha$ and $\beta$, in Eq. (2.1). To determine these parameters, one can use the $L_{2}$ norm to measure the difference between $S_{k-1}(\omega)$ and $P\left[S_{k-1} ; \omega\right]$. 
To linearize the inverse problem, apply the logarithmic transformation to $S_{k-1}(\omega)$ and $P\left[S_{k-1} ; \omega\right]$. Then the objective function is constructed as follows,

$$
J_{\alpha, \beta, c}=\min _{\alpha, \beta, c} \frac{1}{2}\left\|\log \left(S_{k-1}(\omega)\right)-\log \left(P\left[S_{k-1} ; \omega\right]\right)\right\|_{2}^{2},
$$

where $c=\log c_{p}$. The solution of function (A.1) in the least-square sense is

$$
\mathbf{m}=\left(\mathbf{A}^{T} \mathbf{A}\right)^{-1} \mathbf{A}^{T} \mathbf{s},
$$

where $\mathbf{s}=\left[\log S_{k-1}\left(\omega_{1}\right), \log S_{k-1}\left(\omega_{1}+\Delta \omega\right), \log S_{k-1}\left(\omega_{1}+2 \Delta \omega\right), \cdots, \log S_{k-1}\left(\omega_{2}\right)\right]^{T}$, and $\mathbf{m}=$ $[c, \alpha, \beta]^{T}$. The frequency range is $\left[\omega_{1}, \omega_{2}\right]$ and the frequency sampling interval is $\Delta \omega$. The matrix $\mathbf{A}$ is expressed as

$$
\mathbf{A}=\left[\begin{array}{ccc}
1 & \log F_{p}\left(S_{k-1} ; \omega_{1}\right) & \log \left[1-F_{p}\left(S_{k-1} ; \omega_{1}\right)\right] \\
1 & \log F_{p}\left(S_{k-1} ; \omega_{1}+\Delta \omega\right) & \log \left[1-F_{p}\left(S_{k-1} ; \omega_{1}+\Delta \omega\right)\right] \\
\vdots & \vdots & \vdots \\
1 & \log F_{p}\left(S_{k-1} ; \omega_{2}\right) & \log \left[1-F_{p}\left(S_{k-1} ; \omega_{2}\right)\right]
\end{array}\right]
$$

\section{Appendix B: The SVD-HPP algorithm}

When $p$ is less than 1, Eq. (2.12) is generally difficult to be solved because of its nonconvex property. However, if the $L_{p}(0<p<1)$ regularization is transformed into a series of simple $L_{2}$ regularizations [29], to which the existing $L_{2}$ regularization algorithms, such as the SVD method, can be efficiently applied, the non-convex problem will be solved easily. To introduce the algorithm, the cost function (2.12) is rewritten as

$$
J_{\mathbf{r}}=\min _{\mathbf{r}} \mathbf{r}^{T} \operatorname{Dr}-2 \mathbf{r}^{T} \mathbf{L}+\mu\|\mathbf{r}\|_{p}^{p},
$$

where $\mathbf{D}=\mathbf{W}^{T} \mathbf{W}$ and $\mathbf{L}=\mathbf{W}^{T} \mathbf{y}$. Because the matrix $\mathbf{D}$ is symmetric and positive definite, the quadratic optimization problem $\min _{\mathbf{r}} \mathbf{r}^{T} \mathbf{D r}-2 \mathbf{r}^{T} \mathbf{L}$ is equivalent to solving the system of equation $\mathbf{D r}=\mathbf{L}$, which is the normal equations for the least squares problem [44]. The reason why Eq. (2.12) is changed into Eq. (B.1) is to delete the term independent from the variable in Eq. (2.12). Now reparametrize the model $\mathbf{r}$ as $\mathbf{u} \circ \mathbf{v}$ in the case of $p=1$, where $\circ$ is the Hadmard (element-wise) product. The $\mathbf{u}$ and $\mathbf{v}$ can be calculated by the $L_{2}$ regularization, corresponding to the following objective function

$$
J_{\mathbf{u}, \mathbf{v}}=\min _{\mathbf{u}, \mathbf{v}}(\mathbf{u} \circ \mathbf{v})^{T} \mathbf{D}(\mathbf{u} \circ \mathbf{v})-2(\mathbf{u} \circ \mathbf{v})^{T} \mathbf{L}+\mu\left(\mathbf{u}^{T} \mathbf{u}+\mathbf{v}^{T} \mathbf{v}\right) / 2,
$$

where $J_{\mathbf{u}, \mathbf{v}}$ is differentiable and biconvex and its local minimum can be found by using a very simple alternating Tikhonov regularization ( $L_{2}$ regularization) method. Besides, there is a correspondence between the minimums of $J_{\mathbf{u}, \mathbf{v}}$ and $J_{\mathbf{r}}$ [29]. Namely, any local 
minimum $(\hat{\mathbf{u}}, \hat{\mathbf{v}})$ of $J_{\mathbf{u}, \mathbf{v}}$ can provide a global optimal value $\hat{\mathbf{r}}$ of $J_{\mathbf{r}}$. To find such minimum, we rewrite $(\mathbf{u} \circ \mathbf{v})^{T} \mathbf{D}(\mathbf{u} \circ \mathbf{v})$ as $\mathbf{u}^{T}\left(\mathbf{D} \circ \mathbf{v} \mathbf{v}^{T}\right) \mathbf{u}$, and $(\mathbf{u} \circ \mathbf{v})^{T} \mathbf{L}$ as $\mathbf{u}^{T}(\mathbf{v} \circ \mathbf{L})$, and thus,

$$
J_{\mathbf{u}, \mathbf{v}}=\min _{\mathbf{u}, \mathbf{v}} \mathbf{u}^{T}\left(\mathbf{D} \circ \mathbf{v} \mathbf{v}^{T}+\frac{\mu}{2}\right) \mathbf{u}-2 \mathbf{u}^{T}(\mathbf{v} \circ \mathbf{L})+\mu \mathbf{v}^{T} \mathbf{v} / 2 .
$$

Fix $\mathbf{v}$ to solve $\mathbf{u}$ as

$$
\widetilde{\mathbf{u}}=\left(\mathbf{D} \circ \mathbf{v} \mathbf{v}^{T}+\frac{\mu}{2} \mathbf{I}^{\prime}\right)^{-1}(\mathbf{L} \circ \mathbf{v}),
$$

where $\mathbf{I}^{\prime}$ indicates a unit diagonal matrix. Similarly, the $\widetilde{\mathbf{v}}$ can be obtained

$$
\widetilde{\mathbf{v}}=\left(\mathbf{D} \circ \mathbf{u} \mathbf{u}^{T}+\frac{\mu}{2} \mathbf{I}^{\prime}\right)^{-1}(\mathbf{L} \circ \mathbf{u}) .
$$

The forms of Eq. (B.4) and Eq. (B.5) are similar to the conventional Tikhonov regularization solution. To see this, rewrite $(\mathbf{v} \otimes \mathbf{I}) \circ \mathbf{W}^{T}$ and $(\mathbf{u} \otimes \mathbf{I}) \circ \mathbf{W}^{T}$ as $\mathbf{G}_{\mathbf{v}}{ }^{T}$ and $\mathbf{G}_{\mathbf{u}}{ }^{T}$. I indicates an unit row vector and the sign $\otimes$ is a tensor product. Then Eq. (B.4) and Eq. (B.5) can be written as the conventional Tikhonov regularization function

$$
\begin{aligned}
& J_{\widetilde{\mathbf{u}}}=\min _{\widetilde{\mathbf{u}}}\left\|\mathbf{y}-\mathbf{G}_{\mathbf{v}} \widetilde{\mathbf{u}}\right\|\left\|_{2}^{2}+\frac{\mu}{2}\right\| \widetilde{\mathbf{u}} \|_{2}^{2}, \\
& J_{\widetilde{\mathbf{v}}}=\min _{\widetilde{\mathbf{v}}}\left\|\mathbf{y}-\mathbf{G}_{\mathbf{u}} \widetilde{\mathbf{v}}\right\|_{2}^{2}+\frac{\mu}{2}\|\widetilde{\mathbf{v}}\|_{2}^{2} .
\end{aligned}
$$

It's obvious that Eq. (B.6) and Eq. (B.7) represent the conventional Tikhonov regularization. Then we adopt the SVD method to iteratively solve them instead of direct inversion. The book written by [44] contains the SVD implementation of Tikhonov regularization.

The above algorithm is derived in the case of $p=1$. If considering $0<p<1$, the model $\mathbf{r}$ can be reparametrized as $\mathbf{u}_{1} \circ \ldots \circ \mathbf{u}_{k}$. Eq. (B.2) is extended to

$$
J_{\mathbf{u}_{1}, \ldots, \mathbf{u}_{k}}=\min _{\mathbf{u}_{1}, \ldots, \mathbf{u}_{k}}\left(\mathbf{u}_{1} \circ \ldots \circ \mathbf{u}_{k}\right)^{T} \mathbf{D}\left(\mathbf{u}_{1} \circ \ldots \circ \mathbf{u}_{k}\right)-2\left(\mathbf{u}_{1} \circ \ldots \circ \mathbf{u}_{k}\right)^{T} \mathbf{L}+\frac{\mu}{k}\left(\mathbf{u}_{1}^{T} \mathbf{u}_{1}+\ldots+\mathbf{u}_{k}^{T} \mathbf{u}_{k}\right),
$$

where $p=2 / k$. Eq. (B.2) is the special case of Eq. (B.8) when $k=2$. Then we take the derivative of Eq. (B.8) with respect to each parameter, and adopt the SVD method to iteratively solve each $L_{2}$ regularization problem. One can just change the value of parameter $k$ to solve other $L_{p}(0<p<1)$ regularization, if need be.

\section{References}

[1] E. J. Candès and C. Fernandez-Granda, Towards a mathematical theory of super-resolution, Communications on Pure and Applied Mathematics, 67 (2014), 906-956.

[2] M. D. Sacchi and D. R. Velis and A. H. Comínguez, Minimum entropy deconvolution with frequency-domain constraints, Geophysics, 59 (1994), 938-945.

[3] M. D. Sacchi, Reweighting strategies in seismic deconvolution, Geophysical Journal International, 129 (1997), 651-656. 
[4] M. Sajid and D. Ghosh, A fast and simple method of spectral enhancement, Geophysics, 79 (2014), V75-V80.

[5] S. A. M. Oliveira, and W. M. Lupinacci, $L_{1}$ norm inversion method for deconvolution in attenuating media, Geophysical Prospecting, 61 (2013), 771-777.

[6] S. Y. Yuan, S. X. Wang, M. Ma, Y. Z, Ji and L. Deng, Sparse Bayesian learning-based timevariant deconvolution, IEEE Transactions on Geoscience and Remote Sensing, 55 (2017), 6182-6194.

[7] H. L. Chen, J. H. Gao, N. H. Liu and Y. Yang, Multitrace semiblind nonstationary deconvolution, IEEE Geoscience and Remote Sensing Letters, 16 (2019), 1195-1199.

[8] N. D. Hargreaves and A. J. Calvert, Inverse Q filtering by Fourier transform, Geophysics, 56 (1991), 519-527.

[9] Y. H. Wang, A stable and efficient approach of inverse Q filtering, Geophysics, 67 (2002), 657-663.

[10] Y. H. Wang, Quantifying the effectiveness of stabilized inverse Q filtering, Geophysics, 68 (2003), 337-345.

[11] S. D. Stainsby and M. H. Worthington, Q estimation from vertical seismic profile data and anomalous variations in the central North Sea, Geophysics, 50 (1985), 615-626.

[12] S. W. Patton, Robust and least-squares estimation of acoustic attenuation from well-log data, Geophysics, 53 (1988), 1225-1232.

[13] E. Blias, Accurate interval Q-factor estimation from VSP data, Geophysics, 77 (2012), WA149WA156.

[14] C. L. Hackert and J. O. Parra, Improving Q estimates from seismic reflection data using well-log-based localized spectral correction, Geophysics, 69 (2004), 1521-1529.

[15] J. H. Gao, S. L. Yang, D. X. Wang and R. S. Wu, Estimation of quality of factor Q from the instantaneous frequency at the envelope peak of a seismic signal, Journal of Computational Acoustics, 19 (2011), 155-179.

[16] L. L. Wang, J. H. Gao, W. Zhao and X. D. Jiang, Enhancing resolution of nonstationary seismic data by molecular-Gabor transform, Geophysics, 78 (2012), V31-V41.

[17] Y. H. Wang, Inverse Q-filter for seismic resolution enhancement, Geophysics, 71 (2006), V51V60.

[18] I. L. S. Braga and F. S. Moraes, High-resolution gathers by inverse Q filtering in the wavelet domain, Geophysics, 78 (2013), V53-V61.

[19] I. Morozov, M. Haiba and W. B. Deng, Inverse attenuation filtering, Geophysics, 83 (2018), V135-V147.

[20] G. W. Zhang and J. H. Gao, Inversion-driven attenuation compensation using synchrosqueezing transform, IEEE Geoscience and Remote Sensing Letters, 15 (2018), 132-136.

[21] E. J. Candès and M. B. Wakin, Enhancing sparsity by reweighted $L_{1}$ minimization, Journal of Fourier Analysis and Applications, 14 (2008), 877-905.

[22] Z. B. Xu, H. Zhang, Y. Wang and X. Y. Chang, $\mathbf{L}_{1 / 2}$ regularization, Science China Information Sciences, 53 (2010), 1159-1169.

[23] L. L. Wang, Q. Zhao, J. H. Gao, Z. B. Xu, M. Fehler and X. D. Jiang, Seismic sparse-spike deconvolution via Toeplitz-sparse matrix factorization, Geophysics, 81 (2016), V169-V182.

[24] R. Chartrand, Exact reconstruction of sparse signals via nonconvex minimization, IEEE Signal Processing Letters, 14 (2007), 707-710.

[25] M. J. Lai, Y. Y. Xu and W. T. Yin, Improved iteratively reweighted least squares for unconstrained smoothed $l_{q}$ minimization, SIAM Journal on Numerical Analysis, 51 (2013), 927957. 
[26] J. Woodworth and R. Chartrand, Compressed sensing recovery via nonconvex shrinkage penalties, Inverse Problems, 32 (2016), 075004.

[27] H. L. Chen, J. H. Gao and B. Zhang, An adaptive time-varying seismic super-resolution inversion based on Lp regularization, IEEE Geoscience and Remote Sensing Letters, 2020, doi: 10.1109/LGRS.2020.3000339.

[28] M. Elad, Sparse and Redundant Representations: From Theory to Applications in Signal and Image Processing, Springer Publishing Company, Incorporated, 2010.

[29] P. D. Hoff, Lasso, fractional norm and structured sparse estimation using a Hadamard product parametrization, Computational Statistics and Data Analysis, 115 (2017), 186-198.

[30] P. C. Hansen, REGULARIZATION TOOLS: A Matlab package for analysis and solution of discrete ill-posed problems, Numerical Algorithms, 6 (1994), 1-35.

[31] P. C. Hansen, Analysis of discrete ill-posed problems by means of the L-curve, SIAM Review, 34 (1992), 561-580.

[32] G. H. Golub, M. Heath and G. Wahba, Generalized cross-validation as a method for choosing a good ridge parameter, Technometrics, 21 (1979), 215-223.

[33] J. H. Gao, B. Zhang, W. M. Han, J. G. Peng and Z. B. Xu, A new approach for extracting the amplitude spectrum of the seismic wavelet from the seismic traces, Inverse Problems, 33 (2017), 085005.

[34] J. P. Grossman, G. F. Margrave and M. P. Lamoureux, Adaptive, nonuniform Gabor frames from partitions of unity, CREWES Research Report, 14 (2002), 1-10.

[35] Y. L. Quan and J. M. Harris, Seismic attenuation tomography using frequency shift method, Geophysics, 62 (1997), 895-905.

[36] Y. H. Wang and J. Guo, Modified Kolsky model for seismic attenuation and dispersion, Journal of Geophysics and Engineering, 1 (2004), 187.

[37] R. Saab, R. Chartrand and O. Yilmaz, Stable sparse approximations via nonconvex optimization, IEEE International Conference on Acoustics, Speech and Signal Processing, 2008, 3885-3888.

[38] J. K. Pant, W. Lu and A. Antoniou, New improved algorithms for compressive sensing based on $l_{p}$ norm, IEEE Transactions on Circuits and Systems II: Express Briefs, 61 (2014), 198-202.

[39] R. Chartrand and W. T. Yin, Iteratively reweighted algorithms for compressive sensing, IEEE International Conference on Acoustics, Speech and Signal Processing, 2008, 3869-3872.

[40] Z. B. Xu, H. L. Guo, Y. Wang and H. Zhang, Representative of $L_{1 / 2}$ regularization among $L_{q}(0<q \leq 1)$ regularizations: An experimental study based on phase diagram, Acta Automatica Sinica, 38 (2012), 1225-1228.

[41] H. L. Chen, S. Y. Cao, S. Y. Yuan, X. Pan, S. Q. Chen and S. A. Shen, Nonstationary Sparse Reflectivity Inversion with EADTV Regularization, Eage Conference and Exhibition, 2017.

[42] S. Arridge, P. Maass, O. Öktem and C. B. Schönlieb, Solving inverse problems using datadriven models, Acta Numerica, 28 (2019), 1-174.

[43] X. Xu, J. H. Gao, B. Zhang, H. L. Chen and Y. Yang, Multichannel reflectivity inversion with sparse group regularization based on HPPSG algorithm, IEEE Geoscience and Remote Sensing Letters, 17 (2020), 784-788.

[44] C. A. Richard, B. Brian and H. T. Clifford, Parameter Estimation and Inverse Problems (Second Edition), Academic Press, 2011. 